\title{
Giving physical significance to the Hi-GAL^ data: determining the distance of cold dusty cores in the Milky Way ${ }^{\star \star}$
}

D. Russeil ${ }^{1}$, M. Pestalozzi ${ }^{2}$, J. C. Mottram ${ }^{3}$, S. Bontemps ${ }^{4,15}$, L. D. Anderson ${ }^{1}$, A. Zavagno ${ }^{1}$, M. T. Beltrán ${ }^{5}$, J. Bally ${ }^{7}$, J. Brand ${ }^{6}$, C. Brunt ${ }^{3}$, R. Cesaroni ${ }^{5}$, G. Joncas ${ }^{8}$, D. Marshall ${ }^{9}$, P. Martin ${ }^{10}$, F. Massi ${ }^{5}$, S. Molinari ${ }^{2}$, T. Moore ${ }^{11}$, A. Noriega-Crespo ${ }^{12}$, L. Olmi $^{5}$, M. A. Thompson ${ }^{13}$, M. Wienen ${ }^{14}$, and F. Wyrowski ${ }^{14}$

${ }^{1}$ Laboratoire d'astrophysique de Marseille, CNRS/INSU - Université de Provence, 13388 Marseille Cedex 13, France e-mail: delphine.russeil@oamp.fr

2 INAF-Via Fosso del Cavaliere 100, 00133 Roma, Italy

3 School of Physics, University of Exeter, Stocker Road, Exeter, EX4 4QL, UK

${ }^{4}$ CNRS/INSU, Laboratoire d'Astrophysique de Bordeaux, UMR 5804, BP 89, 33271 Floirac cedex, France

5 INAF-Osservatorio Astrofisico di Arcetri, Largo E. Fermi 5, 50125 Firenze, Italy

6 INAF, Istituto di Radioastronomia, Bologna, Italy

7 Center for Astrophysics and Space Astronomy (CASA), Department of Astrophysical and Planetary Sciences, University of Colorado, Boulder, USA

${ }^{8}$ Laval Univ., Observatoire du Mont Megantic, Quebec, Canada

9 CESR, CNRS, Toulouse, France

10 Univ. of Toronto, Canada

11 Astrophysics Research Institute, Liverpool John Moores University, Twelve Quays House, Egerton Wharf, Birkenhead CH41 1LD, UK

12 Spitzer Science Center, California Institute of Technology, Pasadena, CA 91125, USA

13 Center for Astrophysics Research, Science and Technology Research Institute, University of Hertfordshire, Herts AL10 9AB, UK

14 MPIfR-MPG, Bonn, Germany

15 Université de Bordeaux, Observatoire Aquitain des Sciences de l’Univers, Bordeaux, France

Received 1 October 2010 / Accepted 26 November 2010

\begin{abstract}
Context. Hi-GAL, an open time key-project of the Herschel satellite, was awarded 343 hours observing time to carry out a 5-band photometric imaging survey at $70,160,250,350$, and $500 \mu \mathrm{m}$ of a $|b| \leq 1^{\circ}$ wide strip of the Milky Way Galactic plane in the longitude range $-70^{\circ} \leq l \leq 70^{\circ}$. Two $2^{\circ} \times 2^{\circ}$ fields centred at $l=30^{\circ}$ and $l=59^{\circ}$ have been observed with the SPIRE and PACS photometric cameras in parallel mode during the Herschel science demonstration phase (SDP). From the images, compact sources are extracted for which the distance must be established in order to determine their physical properties.

Aims. The aim of this paper is to present the distance determination strategy for the Hi-GAL compact sources. We illustrate this strategy for the two fields at $l=30^{\circ}$ and $l=59^{\circ}$.

Methods. The first step to determine the distance is to establish the LSR (local standard of rest) velocity of each compact source. The kinematic distance is then determined assuming a rotation curve for our Galaxy. To resolve the distance ambiguity for sources within the solar circle, we adopt a multiwavelength approach combining extinction maps, optical, and near infrared images, and velocity information from $\mathrm{NH}_{3}, \mathrm{CO}$ and $\mathrm{HI}$ data. When sources can be kinematically linked to optical $\mathrm{H}$ II regions, the stellar distance of the exciting stars, when known, can be attributed to all linked sources.

Results. In the two $2^{\circ} \times 2^{\circ}$ SDP fields, 2678 compact sources have been identified and listed in the band-merged catalogue. About $93 \%$ of these sources have been assigned a radial velocity and distance.

Conclusions. A multiwavelength approach is necessary to assign the correct velocity to sources (especially when CO spectra have a lot of features) and to determine the distance by solving the distance ambiguity. Also, several Hi-GAL sources seem to be in the interarm region. These sources have to be investigated with dedicated programme to be compared with sources located in the spiral arms.
\end{abstract}

Key words. Galaxy: structure - Galaxy: general - infrared: general - infrared: ISM - H II regions - ISM: clouds

\footnotetext{
* Hi-GAL (Herschel infrared Galactic plane Survey) is a Herschel key-project. Herschel is an ESA space observatory with science instruments provided by European-led Principal Investigator consortia and with important participation from NASA.

$\star \star$ Appendices $\mathrm{A}$ and $\mathrm{B}$ are only available in electronic form at http://www . aanda.org
}

\section{Introduction}

Hi-GAL is an open time key-project of the Herschel satellite that was awarded $343 \mathrm{~h}$ observing time to carry out a 5-band photometric imaging survey at $70,160,250,350$, and $500 \mu \mathrm{m}$ of a $|b| \leq 1^{\circ}$ wide strip of the Milky Way Galactic plane in the longitude range $-70^{\circ} \leq l \leq 70^{\circ}$ (Molinari et al. 2010). The aims of Hi-GAL are to detect the coldest objects in the Galaxy, to study global star-formation in the Milky Way, to define an 
evolutionary sequence for high-mass star formation, and ultimately to compare these results with star-formation gradients in other galaxies. Source extraction was separately performed at all wavelengths, and a band-merged catalogue was compiled (see e.g. Elia et al. 2010; Molinari et al. 2010) containing all sources detected in at least one band (the final version of the catalogue is still under construction and will be published later). An important step is now to establish the distances to the Hi-GAL compact sources. Distance determination enables the computation of physical properties of Hi-GAL sources and allows us to confirm the physical connection between members of large gas complexes and to use them for a general study of the properties of our Galaxy.

The usual method for deriving the distance in the disk of the Milky Way is to assume that the measured radial velocity of the source arises from its differential Galactic rotation. Then, by using a model for the rotation of the Galaxy (e.g. Brand \& Blitz 1993), one can obtain the kinematic distance to the source. The rotation curve models axially symmetric circular orbits, but the true rotation pattern is more complicated.

While the procedure of transforming velocities into distances is straightforward in the outer Galaxy, there is a distance ambiguity for sources in the inner Galaxy. Each velocity measurement leads to two possible distances which correspond to two equidistant points from the point where the line of sight is tangent to the assumed circular orbit of a source. The decision between the near and far distance is only possible using other information, e.g. extinction maps along the same line of sight. Furthermore, velocities that depart from circular rotation will cause errors in the derived distance. Typical velocity deviations of $\sim 10 \mathrm{~km} \mathrm{~s}^{-1}$ (up to $\sim 40 \mathrm{~km} \mathrm{~s}^{-1}$, Brand \& Blitz 1993) are caused by streaming motions (e.g. Burton 1971; Stark \& Brand 1989; Brand \& Blitz 1993; Russeil et al. 2003). This deviation implies a typical distance uncertainty of $\sim 0.9 \mathrm{kpc}$ for $l=59^{\circ}$ and $\sim 0.6 \mathrm{kpc}$ for $l=30^{\circ}$.

We present our procedure to assign heliocentric distances to Hi-GAL sources (in the specific case of this paper, just two HiGAL fields yielded 2678 sources) as well as the first results from the application of this procedure to the two science demonstration phase Hi-GAL fields at $l=30^{\circ}$ and $l=59^{\circ}$.

\section{Distance recommendation pipeline}

We have put into place a pipeline to efficiently determine distances to a large number of sources. Below we explain each step of the pipeline in detail. Figure 1 summarises the four steps of our analysis. The first step is the identification of pointlike sources in Hi-GAL images and the extraction of their photometry. These two tasks are extensively described in Molinari et al. (2010) and Elia et al. (2010). The extraction and photometry of Hi-GAL sources is done through recognition of peaks in the second derivative of the original image in four directions and Gaussian fitting of the sources on top of a plane "plateau" to imitate the bright emission background. Catalogues at the 5 Herschel bands are produced and band-merged (see Elia et al. 2010) to obtain a single list of sources in each Hi-GAL field. To first approximation, a kinematic distance can then be determined after having assigned a radial velocity to each source. Finally, for most of the sources, a distance recommendation has to be given in the catalogues. This recommendation is at least the decision between near- and far- heliocentric (kinematic) distance, and at most a distance determined through association with objects (H II regions, masers with parallax measurment) at known

\section{Source extraction / photometry}

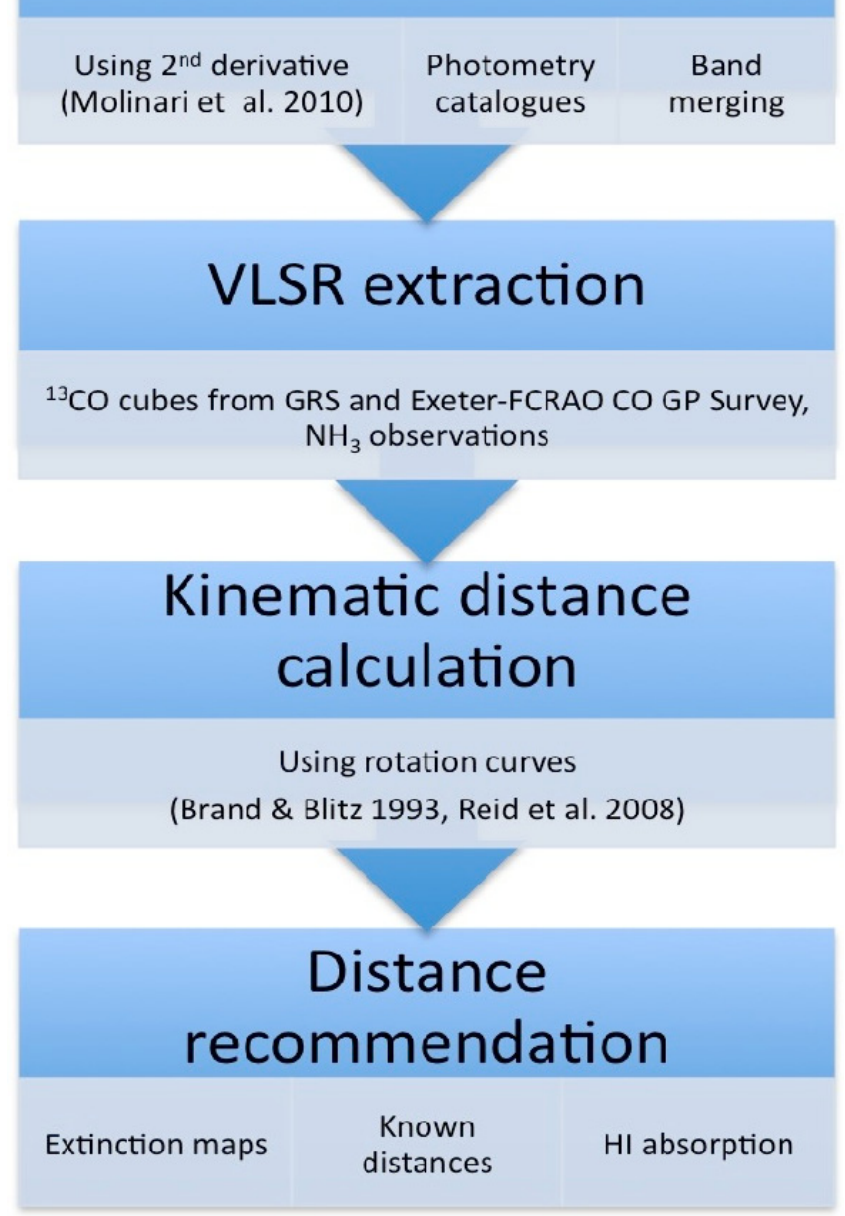

Fig. 1. Distance recommendation pipeline.

distances or other information. In the following subsections we describe these steps in detail.

\subsection{Velocity determination}

\subsubsection{Velocity extraction from $\mathrm{CO}$ data cubes}

We obtain velocities along the line of sight to Hi-GAL sources, where possible, using $\mathrm{CO}$ data observed with the SEQUOIA receiver on the FCRAO $14 \mathrm{~m}$ telescope. In the $l=30^{\circ}$ region we use the ${ }^{13} \mathrm{CO} J=1-0$ data from the BU-FCRAO Galactic Ring Survey (GRS, Jackson et al. 2006) while in the $l=59^{\circ}$ region we use ${ }^{13} \mathrm{CO}$ and $\mathrm{C}^{18} \mathrm{O} J=1-0$ observations obtained as part of the Exeter-FCRAO CO Galactic Plane Survey (Brunt et al., in prep.). These observations have a spatial resolution of $46^{\prime \prime}$ and a spectral resolutions of $0.26 \mathrm{~km} \mathrm{~s}^{-1}$ and $0.16 \mathrm{~km} \mathrm{~s}^{-1}$, respectively. Given the vast number of sources detected in the SDP (science demonstration phase) regions by Hi-GAL, an automated approach to velocity component identification is required. Therefore, line features were identified for each source in the Hi-GAL band-merged catalogue as follows:

1. The spatial pixel containing the source is identified in the molecular line data and a spectrum is formed by integrating spatially within a radius of $60^{\prime \prime}$ of this pixel. 
2. $\sigma_{\text {rms }}$ of the total spectrum is calculated using all velocity channels of the spectrum that are free of emission. The spectrum is first smoothed using a Gaussian kernel with a 3 pixel sigma to avoid sharp noise peaks. The initial estimate of the rms of this smoothed spectrum is calculated using all pixels (i.e. assuming all are emission free). A mask of emission regions is created by identifying each region of the smoothed spectrum $>3 \sigma_{\text {rms }}$ with length $>3$ pixels and adding a mask with twice the length of the region, centred on the region centre. Following the mask creation, the rms is recalculated using the masked smoothed spectrum. The masking and recalculation is repeated until $\sigma_{\text {rms }}$ remains constant, indicating that no new pixels needed to be added to the mask. After this, the $\sigma_{\text {rms }}$ for the spectrum was calculated using the mask and the original unsmoothed spectrum.

3. The channel with maximum intensity is identified and, if above $3 \sigma_{\text {rms }}$, a Gaussian fit is attempted centred at that pixel. While the initial guesses of the line peak, line amplitude, background level, and $\sigma$-width of the Gaussian are input to the fitting, these are all free parameters of the fitting. Where there are multiple Gaussians that need fitting, the background level is a common free parameter for all lines.

4. If the resulting least-squares Gaussian fit has a peak $>3 \sigma_{\text {rms }}$ and a $\sigma$-width $>2$ channels, it is accepted and a residual spectrum calculated, if not, these channels are masked. This 2-channel $\sigma$-width corresponds to thermal profiles with temperatures of $630 \mathrm{~K}$ and $652 \mathrm{~K}$ for the ${ }^{13} \mathrm{CO}$ and $\mathrm{C}^{18} \mathrm{O}$ respectively, indicating that the thermal component of the lines is negligible compared to the line-width due to distance and motion.

5. Steps 3 and 4 are repeated where channels in the masked residual spectrum are $>3 \sigma_{\text {rms }}$, with fitting attempted to the original spectrum using a number of Gaussian components equal to the number of identified lines plus one for the attempted fit, until no channels in the masked residual spectrum fulfil this criterium.

As can be seen from the example shown in Fig. 2, this method is capable of separating partially blended lines. In addition, there is no hard limit placed on the number of components that can be detected, so all components can be identified. This ensures that the maximum amount of information is available for distance determination.

\subsection{2. $\mathrm{NH}_{3}$ observations}

$\mathrm{CO}$ emission is known to be often self-absorbed (optically thick, especially in the Galactic plane) and excited at low densities. Self-absorbtion can be a problem in some of the Hi-GAL SDP lines of sight, precluding the possibility to assign a reliable value for the $V_{\mathrm{LSR}}$ to some of the sources. With this in mind, we observed a subsample of 435 sources from the band-merged catalogue in the NH3 $(1,1)$ transition (Fig. 2) at $23.694495 \mathrm{GHz}$ using the $100 \mathrm{~m}$ Effelsberg, the $30 \mathrm{~m}$ Medicina, and the $20 \mathrm{~m}$ Onsala telescopes. Main beams were 40", 101" and 170", respectively. In all observations rms values of $\approx 40 \mathrm{mK}$ of antenna temperature were reached, which in most cases was enough to detect the main component of the $(1,1)$ transition. All data were reduced either in XS or in the CLASS software from the GILDAS package ${ }^{1}$, with which the spectra were Gaussian fitted to extract the radial velocity of the main hyperfine component. This yielded a general detection rate of about $39 \%$, but the

\footnotetext{
${ }^{1}$ http://www .iram. fr/IRAMFR/GILDAS
}
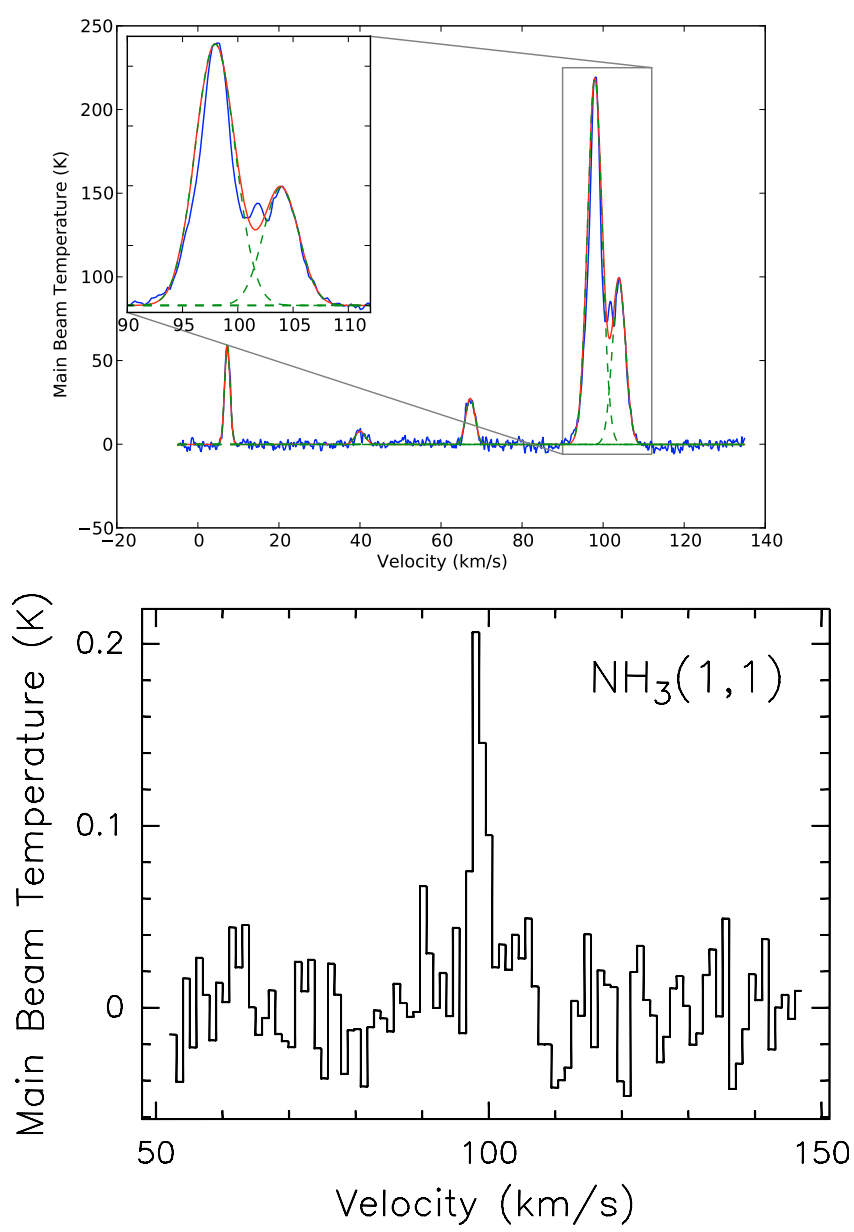

Fig. 2. Example of ${ }^{13} \mathrm{CO}$ spectrum (top, in blue) extracted from the GRS, along with the combined fit to all lines (red) for the Hi-GAL source detected at $l=29.9388^{\circ}, b=0.0167^{\circ}$. The green dashed lines indicate the individual Gaussian components that make up the overall spectrum. The ${ }^{13} \mathrm{CO}$ spectrum exhibits several lines with very different velocities; thanks to the observed $\mathrm{NH}_{3}$ spectrum (bottom) the velocity of the source can be determined $\left(98.41 \mathrm{~km} \mathrm{~s}^{-1}\right)$.

detection rate in the two fields is very different: $49.7 \%$ for $l=30^{\circ}$ and $15.6 \%$ for $l=59^{\circ}$.

\subsubsection{Velocity statistics}

Across the two SDP fields, 2678 Hi-GAL compact sources have been identified and listed in the band-merged catalogue. One $V_{\mathrm{LSR}}$ was assigned to $93.3 \%$ of the sources. As a rule we assigned the $V_{\mathrm{LSR}}$ of the brightest $\mathrm{CO}$ component. This choice is based on the sample of 170 sources with $\mathrm{NH}_{3}$ and $\mathrm{CO}$ velocity. We assume for a source that the $\mathrm{NH}_{3}$ velocity agrees with the ${ }^{13} \mathrm{CO}$ velocity if the departure between them is less than $5 \mathrm{~km} \mathrm{~s}^{-1}$ (the typical molecular cloud velocity dispersion). $85.7 \%$ of the sources have their brightest ${ }^{13} \mathrm{CO}$ component velocity in agreement with the $\mathrm{NH}_{3}$ velocity. In $9.5 \%, 1.8 \%$, and $1.2 \%$ of the cases the velocity agreement is reached with the second, third, and fourth brightest ${ }^{13} \mathrm{CO}$ component respectively. We note that three sources $(1.3 \%)$ clearly disagree between the $\mathrm{NH}_{3}$ and the ${ }^{13} \mathrm{CO}$ velocity.

Conversely, 178 Hi-GAL compact sources are without velocity information (131 and 47 in the $l=59^{\circ}$ and the $l=30^{\circ}$ fields respectively). $63 \%$ of these sources have a flux in one 
band only, $13 \%$ are well detected in the PACS bands, but not or barely in the SPIRE bands, certainly because of the decreasing spatial resolution, and $23 \%$ appear as slightly more intense patches of emission in the filamentary background structures especially at 160 and $250 \mu \mathrm{m}$. The higher number of sources without velocity in the $l=59^{\circ}$ field $\left(12 \%\right.$ and $3 \%$ in the $l=59^{\circ}$ and the $l=30^{\circ}$ fields respectively) can be explained because these sources are probable outer galactic sources farther away than $\sim 15 \mathrm{kpc}$. Indeed, the minimum velocity in GRS data is $-50 \mathrm{~km} \mathrm{~s}^{-1}$, so it does not include the farthest part of the outer arm. This is illustrated by the VLA Galactic Plane Survey ${ }^{2}$ (Stil et al. 2006) HI longitude-velocity plot (Fig. A.2), which shows clear features with a velocity more negative than $-50 \mathrm{~km} \mathrm{~s}^{-1}$.

We can estimate in the SPIRE bands the extragalactic background contamination from the number counts of galaxies (Clements et al. 2010). For the typical detection level ( $\sim 1 \mathrm{Jy}$ and $\sim 0.35 \mathrm{Jy}$ for $l=59^{\circ}$ and the $l=30^{\circ}$ fields in the SPIRE bands respectively) we can estimate for $250 \mu \mathrm{m}$ to $500 \mu \mathrm{m} \sim 4$ galaxies in the $l=30^{\circ}$ field, while for $l=59^{\circ}$ field $\sim 12$ galaxies at $250 \mu \mathrm{m}$ and $\sim 4$ galaxies at $350 \mu \mathrm{m}$ and $500 \mu \mathrm{m}$ are expected. We therefore conclude that the contamination of Hi-GAL sources in the SDP fields by extragalactic objects is negligible.

Finally, 708 sources $(26.5 \%)$ have a unique velocity determination (only one line detected in the spectrum) from $\mathrm{NH}_{3}, \mathrm{C}^{18} \mathrm{O}$, or ${ }^{13} \mathrm{CO}$.

\subsection{Distance calculation}

Kinematic distances were calculated for all sources with an assigned $V_{\text {LSR }}$ using the Brand \& Blitz (1993) rotation curve, with no correction for probable velocity departures because these were not evaluated. We favour the Brand \& Blitz (1993) rotation curve instead of the more recent rotation curve by Reid et al. (2009). We support our choice with the fact that the Reid et al. (2009) rotation curve is based only on parallax of sources in the northern hemisphere, while Hi-GAL will survey a significant portion of the fourth quadrant too.

The Reid et al. (2009) rotation curve is based on a maser parallax in 18 star-forming regions located only in the first and second Galactic quadrants. Reid et al. (2009) adopt a flat rotation curve (with velocity $254 \mathrm{~km} \mathrm{~s}^{-1}$ and $R_{0}$ of $8.4 \mathrm{kpc}$ ) and incorporate a significant revision to the standard solar motion currently used to adjust velocities to the LSR; they also apply a velocity correction (which partially counteracts the solar motion revision) assumed to be applicable to high-mass young stars. As the two SDP fields are in the first Galactic quandrant, they are well suited to quantify the influence of the rotation curve choice to the kinematic distance determination. The difference between the two curves is shown in Fig. 3. For the $l=30^{\circ}$ direction the distance discrepancy between both rotation curves is more critical near the tangent point, while for $l=59^{\circ}$ there is a systematic shift between both velocity-distance curves. But the typical distance uncertainty calculated by Reid et al. (2009) for a $7 \mathrm{~km} \mathrm{~s}^{-1}$ velocity uncertainty (clouds velocity dispersion typical value) are $0.69 \mathrm{kpc}$ and $0.96 \mathrm{kpc}$ for $l=30^{\circ}$ and $l=59^{\circ}$ respectively. Consequently for both direction the distance from the Brand \& Blitz (1993) rotation curve can be considered within the error bar of the Reid et al. (2009) distance, except between 6 and $8 \mathrm{kpc}$ (around the tangent point) for $l=30^{\circ}$ and farther away than $11.5 \mathrm{kpc}$ for $l=59^{\circ}$.

In addition, while the maser parallax distances are very promising to trace the rotation curve and probe the velocity

2 VGPS, http://www.ras.ucalgary.ca/VGPS/.
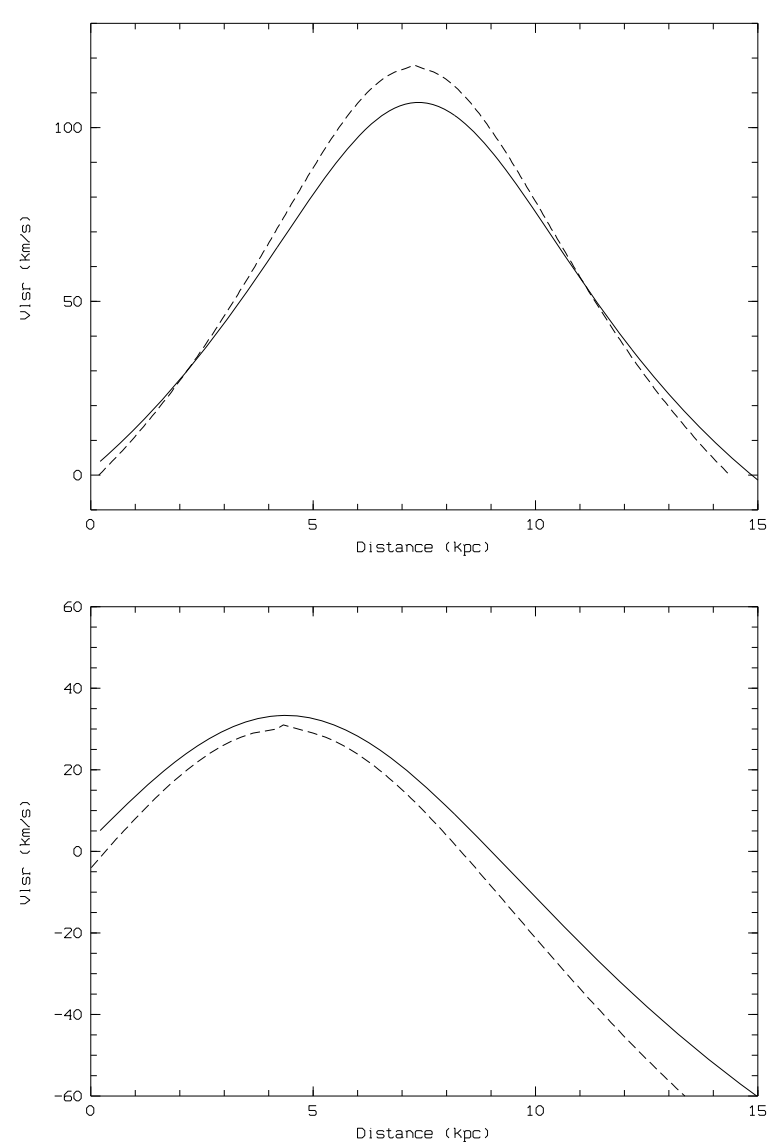

Fig. 3. Distance-velocity relationship for $l=30^{\circ}(t o p)$ and $l=59^{\circ}($ bottom) derived from the Brand \& Blitz (1993) rotation curve (continuous curve) and the Reid et al. (2009) rotation curve (dashed curve).

departures, the Reid et al. (2009) results have still to be confirmed (McMillan \& Binney 2010). From the same data Bovy et al. (2009) confirm the lag of $15 \mathrm{~km} \mathrm{~s}^{-1}$ but found a velocity for the Sun of $244 \mathrm{~km} \mathrm{~s}^{-1}$. In parallel, McMillan \& Binney (2010) underline that the sources that provide strongest statistical support for this $15 \mathrm{~km} \mathrm{~s}^{-1}$ lag are found near the Sun and that similar results can be obtained if the Sun is assumed to circulate faster by $17 \mathrm{~km} \mathrm{~s}^{-1}$. Finally, Baba et al. (2009) show from simulations that there is no clear tendency that star-forming gas moves slower than the galactic rotation, and they found the peculiar velocities to be randomly oriented.

Conversely the Brand \& Blitz (1993) rotation curve is more representative of the general rotation of our Galaxy because it is built on a large sample of complexes located in all four Galactic quadrants and combines stellar distances and $\mathrm{HI} / \mathrm{CO}$ tangent distances.

Accordingly the usual method used to determine the kinematic distance is as follows:

1. The angular velocity is determined from the measured $V_{\mathrm{LSR}}$ via the relation (e.g. Brand \& Blitz 1993):

$\omega=\omega_{\mathrm{O}}+\frac{V_{\mathrm{LSR}}}{R_{\mathrm{O}} \sin (l) \cos (b)}$,

where $\omega_{\mathrm{O}}$ and $R_{0}$ are the adopted solar angular velocity and galactocentric distance. This relation assumes circular uniform velocity for the object. 
2. For the angular velocity $\omega$, the galactocentric distance of the object is determined by inverting (numerically) the chosen rotation curve.

3. The heliocentric distance is obtained from the well known equation (e.g. Brand \& Blitz 1993)

$$
d^{2} \cos ^{2}(b)-\left(2 R_{0} \cos (b) \cos (l)\right) d+\left(R_{0}^{2}-R^{2}\right)=0 \text {. }
$$

This relation gives two solutions for the distance of an object within the solar circle, while only one solution will be physically acceptable for sources outside the solar circle.

\subsection{Distance recommendation: resolution of the kinematic distance ambiguity}

Because both SDP Hi-GAL fields lie in the inner Galaxy, the kinematic distance for most of the compact sources identified there suffers from the near/far ambiguity. To solve the distance ambiguity problem we follow a multiwavelenth approach. The first step is based on extinction features. For both fields the extinction map is derived from the publicly available $2 M A S S$ point source catalogue (see Schneider et al. 2010, for details). These maps have a resolution of $2^{\prime}$ with a $1^{\prime}$ pixel size. From the extinction maps (Fig. 4) as described in Schuller et al. (2009) it is possible to recognize that some structures account for the detected extinction and are therefore probably at the near kinematic distance. For the $l=30^{\circ}$ and $l=59^{\circ}$ fields, the large scale features in the extinction maps seem to correlate with the brightest patches in Hi-GAL data at $250 \mu \mathrm{m}$, suggesting that most of the (cold) dust emission comes from the near side of the Galaxy.

As a second step, by morphologically matching the extinction features with the ${ }^{13} \mathrm{CO}$ emission datacube, we determined the most probable velocity of the extinction features. The assignment of $V_{\text {LSR }}$ to large regions of the extinction map considerably accelerates the process of assigning a near/far distance to a large number of sources because this helps us to decide which HiGAL sources are linked to the extinction features. We complete our extinction approach by looking at the position of sources in the extinction and emission features seen on Spitzer $8 \mu$ m images and, when possible, to $\mathrm{H} \alpha$ or optical images.

As a third step, we use the clear association (close relative position both in space and velocity) of some Hi-GAL sources with known H II regions for which distances have been determined or for which at least the distance ambiguity is solved.

For H II regions the distance ambiguity can be solved using HI emission/absorption (HIE/A) and self-absorption (HISA) methods (e.g. Anderson \& Bania 2009) or $\mathrm{H}_{2} \mathrm{CO}$ or OH absorption line velocity (e.g. Sewilo et al. 2004). The HIE/A method (e.g. Kuchar \& Bania 1994; Fish et al. 2003; Kolpak et al. 2003; Anderson \& Bania 2009) relies on absorption of HI gas distributed along the line of sight between the H II region and the observer. Since HI emits at all allowed velocities and the continuum emission of $\mathrm{H}$ II regions is broadband, $\mathrm{HI}$ absorption can be detected in principle for any $\mathrm{H}$ II region. HI absorption detected beyond the velocity of the $\mathrm{H}$ II region implies that the $\mathrm{H}$ II region lies at the far distance; $\mathrm{HI}$ absorption detected up to, but not exceeding the $\mathrm{H}$ II region velocity implies that the $\mathrm{H}$ II region lies at the near distance. HI self-absorption (e.g. Knapp 1974; Jackson et al. 2002; Busfield et al. 2006; Anderson \& Bania 2009; Roman-Duval et al. 2009) relies on absorption of cold HI associated with molecular gas at the near distance from warmer $\mathrm{HI}$ at the far distance. In this scenario, the absorption is caused because the cold and warm HI share the same velocity. The detection of $\mathrm{HI}$ absorption at the molecular gas velocity implies
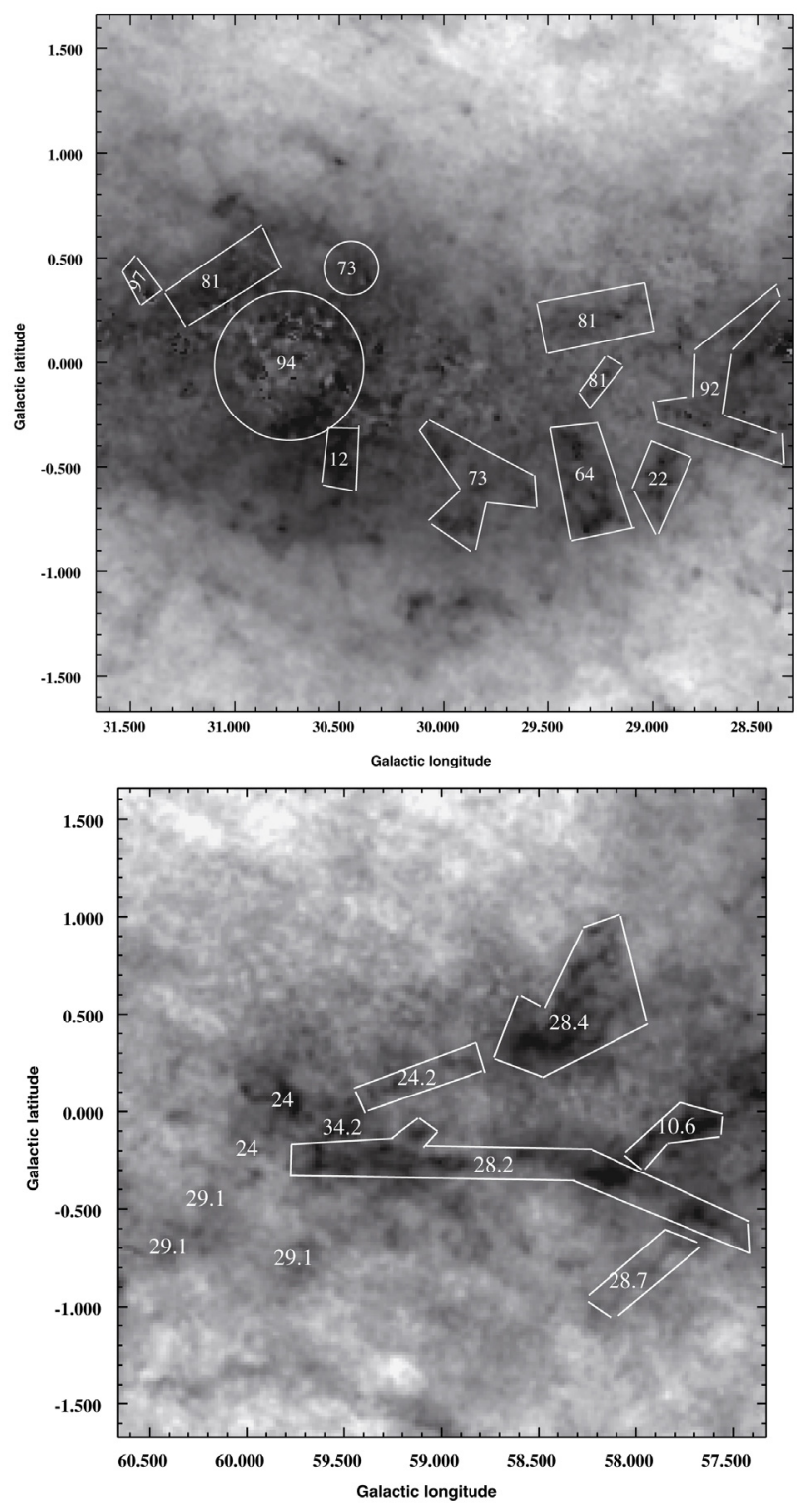

Fig. 4. Extinction maps for $l=30^{\circ}$ (top) and $l=59^{\circ}$ (bottom) fields (Bontemps, priv. comm.). Numbers are ${ }^{13} \mathrm{CO}$ velocities of the extinction features at the position of the number or for the delimited areas. The extinction range from $4.3 \mathrm{mag}$ (white pixels) to $\geq 32.3$ mag (black pixels) for the $l=30^{\circ}$ field and from $2 \mathrm{mag}$ (white pixels) to $15 \mathrm{mag}$ (black pixels) for the $l=59^{\circ}$ field.

that the molecular gas is at the near distance; the absence of absorption at the molecular gas velocity implies that the molecular gas is at the far distance. We can note that HI method results must be considered carefully as demonstrated for the $\mathrm{H}$ II region Sh2-86 (Table B.2), for which a stellar distance of $2.3 \mathrm{kpc}$ is established, while HI emission/absorption analysis suggests a far kinematic distance.

In addition to solve the distance ambiguity for individual objects, assuming that stars form in Giant Molecular Clouds (GMC), we group H II regions, molecular clouds, and infrared dark clouds (IRDCs) into star-forming complexes, on the basis of a close relative position both in space and velocity with GMCs (e.g. Dame et al. 1986; Jacq et al. 1988). Objects are grouped together if their position and velocity fall within the spatial extent and velocity width of the GMC. Sometimes the grouping 
is exclusively composed of $\mathrm{H}$ II regions or molecular clouds; in these cases the grouping is less certain.

We can then use individual kinematic distances and/or stellar (photometric or spectro-photometric) distances of H II regions' exciting stars (or maser distance of star-forming regions) to determine the most probable distance of the complex, and thus assign all sources in the complex a common near/far solution. The distance ambiguity solution for Hi-GAL sources can then be established either from their association to an $\mathrm{H}$ II region or to a star-forming complex. In this way we have compiled velocities and near/far distance information (Tables B.1 and B.2) for H II regions, molecular clouds and IRDCs in the $l=30^{\circ}$ and $l=59^{\circ}$ fields. Also, the identification of star-forming complexes allow us to have a general picture of the velocities and distances expected in the field of view.

The distance recommendation scheme is then:

1. The Hi-GAL source is associated with an H II region:

(a) If the H II region has a known stellar (parallax) distance, this distance is assigned to the Hi-GAL source.

(b) If the H II region has a well established near, far, or tangent distance ambiguity solution: the same near, far, or tangent solution is adopted for the kinematic distance calculation of the Hi-GAL source.

2. The Hi-GAL source is associated with a complex:

(a) If a stellar (parallax) distance is assigned to the complex, this distance is assigned to the Hi-GAL source.

(b) If the near, far, or tangent distance ambiguity is solved for the complex, the same near, far, or tangent solution is adopted for the kinematic distance calculation of the Hi-GAL source.

3. The Hi-GAL source is associated with an extinction feature: the near distance is favoured.

Some Hi-GAL sources fullfil several of these conditions. Usualy the conditions agree for the same distance solution. If they do not, we favour the distance (by order of priority) given by the items 1a, 2a, 3, 2b, 1b.

\section{Results}

\subsection{Distances in the $I=59^{\circ}$ direction}

The $l=59^{\circ}$ field is dominated by the optical H II region Sh286 (27.9 $\mathrm{km} \mathrm{s}^{-1}$, Fich et al. 1990), which appears as a hole in the atomic gas distribution from the VGPS at velocities between 26 and $31 \mathrm{~km} \mathrm{~s}^{-1}$ (Billot et al. 2010). Sh2-86, excited by the star cluster NGC 6823, belongs to the same complex as Sh2-87, Sh2-88, Sh2-89, and Sh2-90 (Brand \& Blitz 1993) and the stellar association VulOB1. Distance determinations for the H II regions and their exciting stars are between 1.5 and $3.2 \mathrm{kpc}$ (e.g. Massey et al. 1995; Hoyle et al.2003; Guetter et al. 1992, Peña et al. 2003). Recent distance determination based on maser parallax give a distance of $2.16_{-0.09}^{+0.10} \mathrm{kpc}$ for the maser G59.7+0.1 (Xu et al. 2009), while a distance of $1.95 \pm 0.25 \mathrm{kpc}$ is estimated with the extinction method (Marshall et al. 2006, Fig. 6). We adopt a mean distance of $2.1 \mathrm{kpc}$ for Sh2-86, which places it in the near part of the Sagittarius arm.

The Hi-GAL sources in this field mainly have velocities between 20 and $50 \mathrm{~km} \mathrm{~s}^{-1}$ and -15 and $0 \mathrm{~km} \mathrm{~s}^{-1}$ (Fig. 3). For sources with negative velocities and velocities near $0 \mathrm{~km} \mathrm{~s}^{-1}$ there is no distance ambiguity problem. The sources with velocities $<-40 \mathrm{~km} \mathrm{~s}^{-1}$ are around $13.4 \mathrm{kpc}$ and the others with velocities between -15 and $0 \mathrm{~km} \mathrm{~s}^{-1}$ are around $9 \mathrm{kpc}$. We note

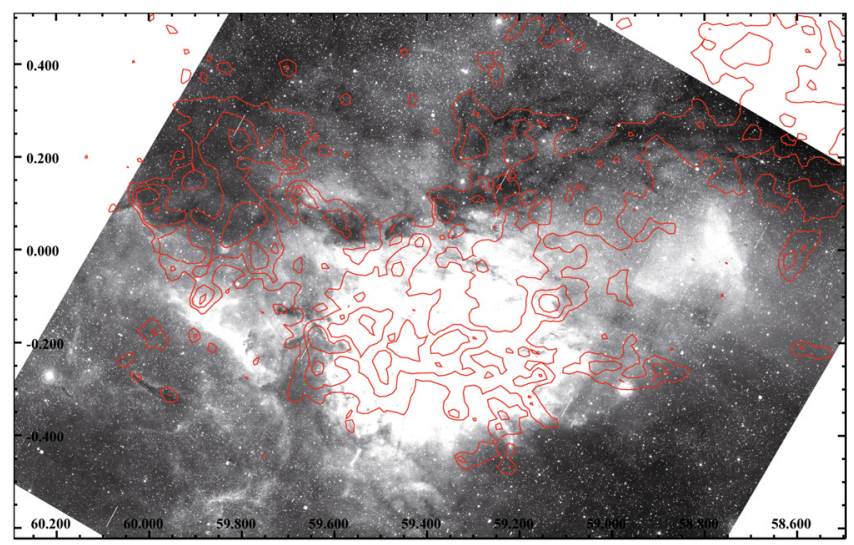

Fig. 5. $\mathrm{H} \alpha$ image (galactic coordinates) of Sh2-86 (IPHAS) with superimposed Hi-GAL $500 \mu \mathrm{m}$ isocontours. We note the very good correlation between the dark lanes and the $500 \mu \mathrm{m}$ emission allowing us to favour the near distance for most of the $500 \mu \mathrm{m}$ filaments.

that a few sources have forbidden velocities (higher than the tangent velocity of $34 \mathrm{~km} \mathrm{~s}^{-1}$ ). The only way to assign these sources a distance is to take the tangent point distance.

At $70 \mu \mathrm{m}$, the Hi-GAL image shows pillar-like structures to the north-east (Galactic coordinates) of Sh2-86 and inside Sh286 and shows extended filaments to the south-west (Galactic coordinates) of Sh2-86. All these features are bathed in the ionised emission of Sh2-86 as seen in $\mathrm{H} \alpha$, but some pillar-like structures point towards stars of VulOB1 other than NGC6823 (Billot et al. 2010). The filamentary features are probably associated with Sh2-86 or at least can be put at the near distance because they correspond to extinction features in the extinction map, the $\mathrm{H} \alpha$ image (Fig. 5), and the DSS-red image.

To help assign distances to sources with velocities between 18 and $34 \mathrm{~km} \mathrm{~s}^{-1}$ the line of sight extinction map towards the $l=59^{\circ}$ field (Av) for distances between 1.7 and $2.2 \mathrm{kpc}$ has been constructed (Fig. 6) using the method of Marshall et al. (2009). This method performs a comparison of observed stars (2MASS, Skrutskie et al. 2006, and GLIMPSE (Benjamin et al. 2003) against the predictions of a three dimensional model of the Galactic stellar populations (Robin et al. 2003). The resulting extinction is that which creates a colour distribution of the modelled stars that agrees with the observed distribution. The Galaxy model provides the intrinsic colour of stars and their probable distances, so that the near-infrared colour excess, and hence the extinction, may be calculated and its distance evaluated. The extinction feature between $59^{\circ}$ and $60^{\circ}$ corresponds to Sh2-86 (Fig. 6). We can note that other extinction features are located in the same distance range. In this way the majority of the sources with a velocity between 18 and $30 \mathrm{~km} \mathrm{~s}^{-1}$ are most probably associated with Sh2-86 (at $2.1 \mathrm{kpc}$ ) because they appear located in the photodissociation region, in dark filaments surrounding Sh2-86 as seen on the $8 \mu \mathrm{m}$ Spitzer image, or found in the direction of extinction features (Figs. 4 and 6). A few sources with velocities between 22 and $35 \mathrm{~km} \mathrm{~s}^{-1}$ have been placed at the far distance since there are no associated extinction features.

Figure 7 illustrates the near/far distance solution we propose. At the position of Hi-GAL sources we listed their velocity. If we focus on sources that can be placed at the near distance, we note that these sources are either associated with the H II regions Sh286 (black numbers) and the radio H II regions (listed Table B.2) "Wat58.77+0.65" and "Wat59.60+0.92" (red numbers around these positions), or with no H II region (other red sources). This 


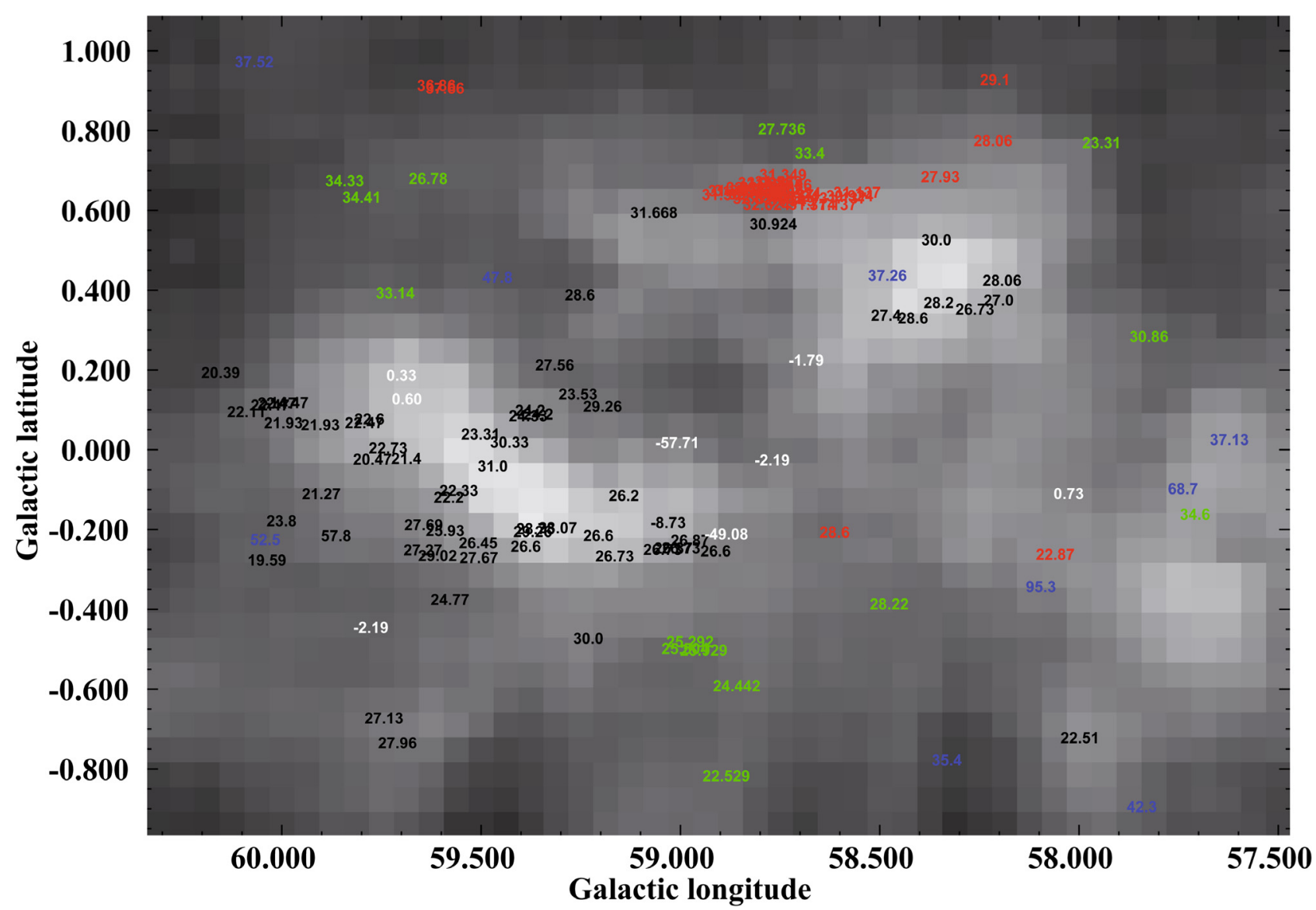

Fig. 6. Extinction map (Av) for distances between 1.7 and $2.2 \mathrm{kpc}$. The extinction values range from $\sim 0.37$ mag (black pixels) to $\sim 1.86 \mathrm{mag}$ (white pixels). Each number is the velocity of a source. The colours correspond to: black: sources most probably associated with Sh2-86 (2.1 kpc), red: near distance $(\sim 3 \mathrm{kpc})$, but not associated with Sh2-86, white: objects with negative velocity (no distance ambiguity, $\sim 13.5 \mathrm{kpc}$ ) or velocity close to $0 \mathrm{~km} \mathrm{~s}^{-1}$ (far distance favoured, $\sim 9.5 \mathrm{kpc}$ ), Blue: objects with forbidden velocity; tangent distance adopted $(\sim 4.3 \mathrm{kpc}$ ), and green: far distance $(\sim 6 \mathrm{kpc})$.

underlines the importance of including evidence of an association between Hi-GAL sources and $\mathrm{H}$ II regions in the distance determination process, which can help to separate sources that are probably involved in triggering star-formation processes from those that are not. In parallel, three infrared bubbles from Churchwell et al. (2006) are present in the field and show a clear $70 \mu \mathrm{m}$ counterpart. These bubbles are primarily formed by hot massive young stars because Deharveng et al. (2010) estimate that up to $93 \%$ of the bubbles enclose H II regions. They are potential sites of trigerring star-formation (Deharveng et al. 2010). Thanks to their association with Hi-GAL sources we can determine the distances of two of these bubbles: N124 $\left(l, b=58.605^{\circ}, 0.638^{\circ}\right)$ and $\mathrm{N} 125$ (corresponding to the H II region "Wat58.77+0.65") can be placed at 5.6 and $4.3 \mathrm{kpc}$ respectively. For $\mathrm{N} 126\left(l, b=59.606^{\circ}, 0.330^{\circ}\right)$ no Hi-GAL source has been extracted because of its diffuse morphology.

\subsection{Distances in the $I=30^{\circ}$ direction}

The $l=30^{\circ}$ field is dominated by two luminous massive starforming complexes: W43 (92.02 $\mathrm{km} \mathrm{s}^{-1}$, Quireza et al. 2006) and G29.96-0.02 (95.9 $\mathrm{km} \mathrm{s}^{-1}$, Kim \& Koo 2001). As they are not optically visible, it is not possible to optically identify the exciting stars and thus no spectro-photometric distance can be determined. In the field, there are also three small optical H II regions Sh2-65, Sh2-66 and Sh2-67 (with velocities $49.3 \mathrm{~km} \mathrm{~s}^{-1}$,
$46.7 \mathrm{~km} \mathrm{~s}^{-1}$ and $18.3 \mathrm{~km} \mathrm{~s}^{-1}$ respectively, Fich et al. 1990) with known stellar distances (Forbes 1989; see Table B.1).

The velocity of W43 and G29.96-0.02 are close to the tangent velocity, which makes it difficult to solve the distance ambiguity. For W43 HIE/A, HISA and absorption lines all agree to imply the near distance solution. In addition W43 belongs to complex 8 (Table B.1), which is a large star-forming complex including infrared dark clouds and other $\mathrm{H}$ II regions for which we adopt the near distance. For G29.96-0.02 the distance determination is more difficult to establish. G29.96-0.02 could belong to the same star-forming complex as W43 owing to its position and velocity, but HI data from several references suggest the far distance. The absorption lines along the line of sight of G29.96-0.02 (Sewilo et al. 2004) show velocities similar to the ones observed towards H II regions of complex 8, but do not allow to clearly solve the distance ambguity. Pratap et al. (1999) give arguments from stellar near-infrared data and extinction considerations for choosing the near distance. In addition, at large scales, HI appears in absorption, which suggests the near distance (Nguyen et al., in prep.). While we wait for a definitive distance determination, we adopt the near distance for G29.96-0.02 from the maser parallax method.

The Hi-GAL sources mainly have velocities (Fig. 9) between 90 and $105 \mathrm{~km} \mathrm{~s}^{-1}$, showing that they are associated with either W43 or G29.96-0.02. In addition to the H II regions, a large circular molecular structure with a radius of $\sim 0.79^{\circ}$, centred at $(l, b)=\left(30.56^{\circ},-0.057^{\circ}\right)$, is seen in ${ }^{13} \mathrm{CO}$ with velocities 


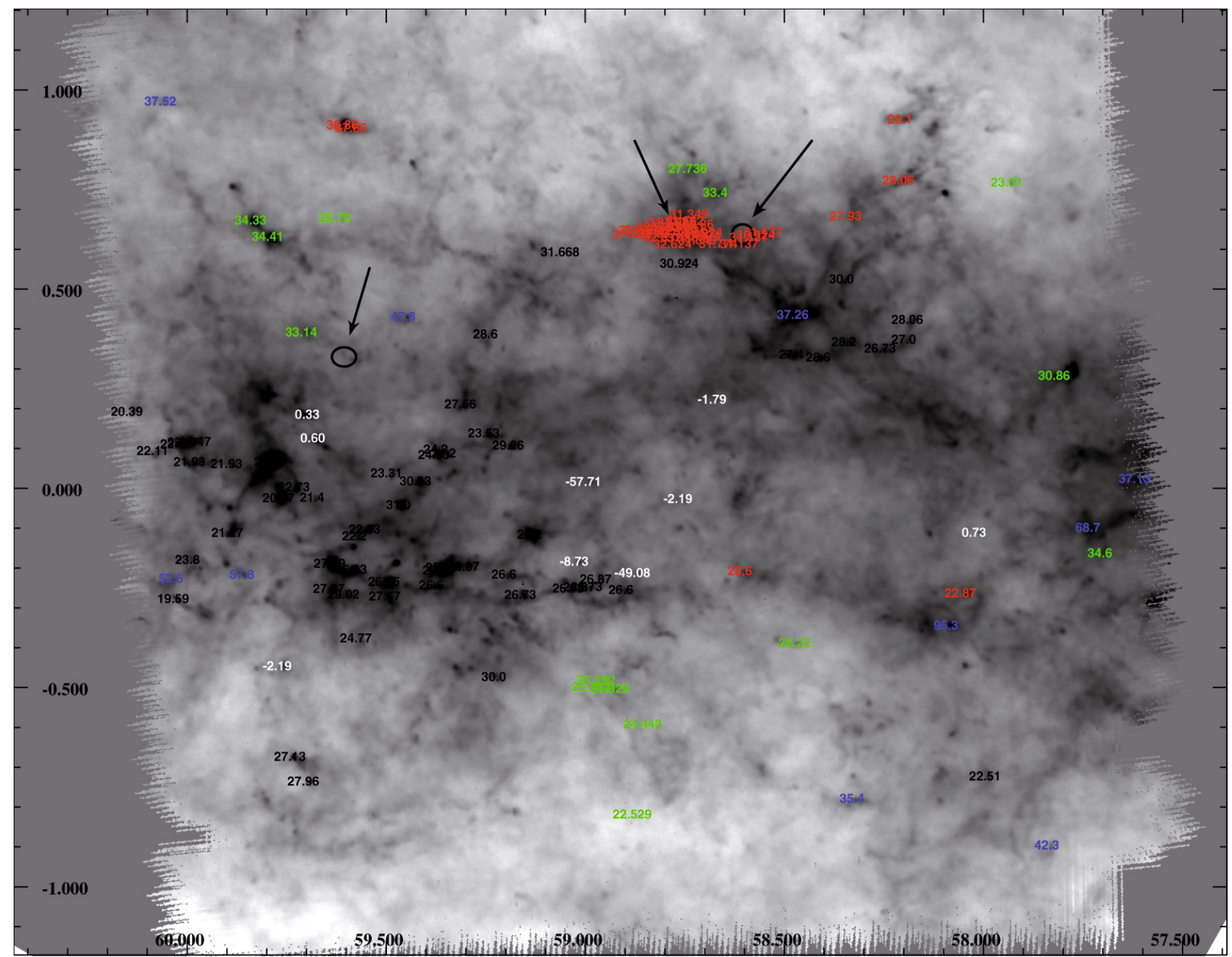

Fig. 7. Hi-GAL $500 \mu \mathrm{m}$ image (the coordinates are galactic coordinates) of the $l=59^{\circ}$ field. Each number is the velocity of a source. The colours correspond to: black: sources most probably associated with Sh2-86 $(2.1 \mathrm{kpc})$, red: near distance $(\sim 3 \mathrm{kpc})$, but not associated with Sh2-86, white: objects with negative velocity (no distance ambiguity, $\sim 13.5 \mathrm{kpc}$ ) or velocity close to $0 \mathrm{~km} \mathrm{~s}^{-1}$ (far distance favoured, $\sim 9.5 \mathrm{kpc}$ ), blue: objects with forbidden velocity; tangent distance adopted $(\sim 4.3 \mathrm{kpc})$, and green: far distance $(\sim 6 \mathrm{kpc})$. The black ellipses pointed out by arrows are the three infrared bubbles from Churchwell et al. (2006) discussed in the text.

between 70 to $85 \mathrm{~km} \mathrm{~s}^{-1}$ (Fig. A.1). Several Hi-GAL sources and features are seen towards this structure; it is therefore important to establish its distance. Absorption lines between 74 and $89 \mathrm{~km} \mathrm{~s}^{-1}$ are frequently noted (Table B.1) towards H II regions both at the far and the near distance and with $l>29.8^{\circ}$. This suggests that the near distance $(\sim 5 \mathrm{kpc})$ can be assigned to this molecular structure, leading to a physical radius of $\sim 69 \mathrm{pc}$. No HI counterpart is detected for this feature and it is not listed in the Heiles (1979) and Ehlerová \& Palous (2005) HI shells catalogues.

A few sources have velocities higher than $110 \mathrm{~km} \mathrm{~s}^{-1}$ (higher than the tangential velocity, but within the typical velocity range due to the streaming motion for most of the sources); we assign them to the tangent point distance. Hi-GAL sources below $l=29.60^{\circ}$ with velocities between 87 and $105 \mathrm{~km} \mathrm{~s}^{-1}$ can be split into near and far sources on the basis of their association with extinction features and star-forming complexes. Finally, a few sources have velocities around $10 \mathrm{~km} \mathrm{~s}^{-1}$; some of them have been put at the near distance. It is not surprising to find sources this close because in the field Sh2-67 is located at a stellar distance of $0.5 \mathrm{kpc}$ (Forbes 1989). Figure 8 illustrates the near/far distance solution we propose.
Six infrared bubbles (N49, N50, N51, N52, N53, and N54) from Churchwell et al. (2006) are present in this field and show a clear $70 \mu \mathrm{m}$ counterpart except for N51 $\left(l, b=29.158^{\circ}\right.$, $\left.-0.262^{\circ}\right)$. All, except for N51 are classified as H II region by Deharveng et al. (2010). The velocities (ionised gas) of these bubbles given in Deharveng et al. (2010) are $90.6 \mathrm{~km} \mathrm{~s}^{-1}$, $67.7 \mathrm{~km} \mathrm{~s}^{-1}, 91.6 \mathrm{~km} \mathrm{~s}^{-1}, 43.6 \mathrm{~km} \mathrm{~s}^{-1}$, and $104.7 \mathrm{~km} \mathrm{~s}^{-1}$ for $\mathrm{N} 49, \mathrm{~N} 50, \mathrm{~N} 52, \mathrm{~N} 53$, and N54 respectively. We can determine the distance to these bubbles. N52 $\left(l, b=30.749^{\circ}\right.$, $\left.-0.019^{\circ}\right)$ corresponds to $\mathrm{W} 43(5.8 \mathrm{kpc})$ and $\mathrm{N} 49\left(l, b=28.827^{\circ}\right.$, $\left.-0.229^{\circ}, 5.5 \mathrm{kpc}\right)$ is a well known triggering star-forming region (Zavagno et al. 2010). N53 $\left(l, b=31.157^{\circ},-0.145^{\circ}\right)$ and $\mathrm{N} 54\left(l, b=31.164^{\circ},+0.292^{\circ}\right)$ are located in the direction of the molecular annulus, but their velocities show that they do not belong to it. They can be associated with complexes 13 and 10 (Table B.1) and then placed at $11.3 \mathrm{kpc}$ and $7.3 \mathrm{kpc}$ respectively. $\mathrm{N} 50\left(l, b=29.001^{\circ},+0.097^{\circ}\right)$ corresponds to the Hi-GAL source higal-129-55. But the velocity adopted for this Hi-GAL source $\left(97.06 \mathrm{~km} \mathrm{~s}^{-1}\right.$ ) from ${ }^{13} \mathrm{CO}$ disagrees with the ionised gas velocity. The ionised gas velocity corresponds to another, less intense, component of the ${ }^{13} \mathrm{CO}$ profile. This underlines the importance to have an $\mathrm{NH}_{3}$ and/or $\mathrm{C}^{18} \mathrm{O}$ velocity. $\mathrm{N} 51$ is a peculiar case 


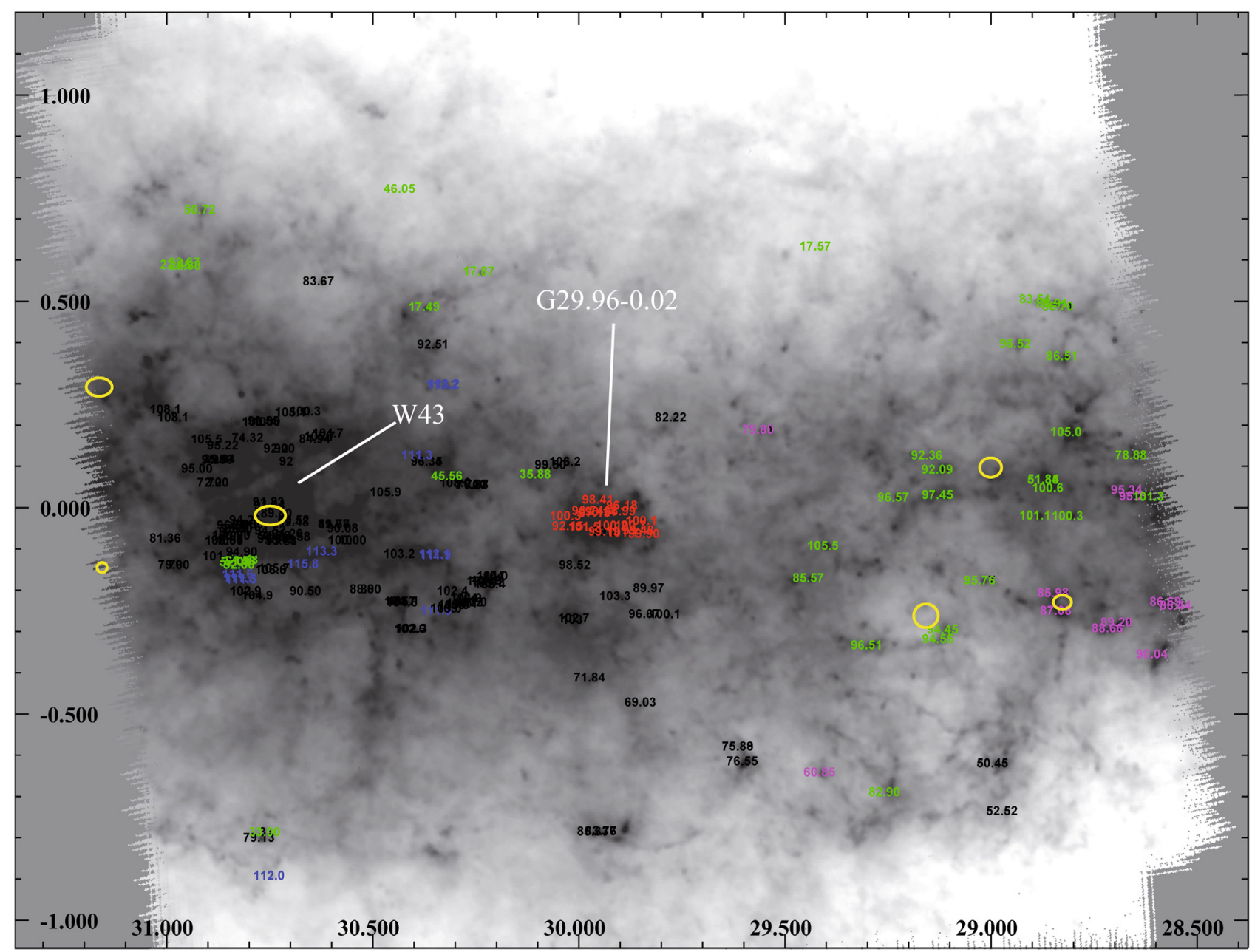

Fig. 8. Hi-GAL $500 \mu \mathrm{m}$ image (the coordinates are Galactic coordinates) of the $l=29^{\circ}$ field. The colours correspond to: black: probable assocation with W43 for sources around $94 \mathrm{~km} \mathrm{~s}^{-1}(\sim 5.8 \mathrm{kpc})$ and association with Sh2-65 (around $l, b=29.15^{\circ},-0.75^{\circ}$; see Fig. A.1) for sources around $51 \mathrm{~km} \mathrm{~s}^{-1}(3.3 \mathrm{kpc})$, blue: tangent distance $(\sim 7.4 \mathrm{kpc})$, green: far or tangent distance (e.g. $\sim 12.4 \mathrm{kpc}$ for sources $\sim 20 \mathrm{~km} \mathrm{~s}{ }^{-1}, \sim 7.5 \mathrm{kpc}$ for sources $\left.\sim 95 \mathrm{~km} \mathrm{~s}^{-1}\right)$, magenta: Near distance $(\sim 5.3 \mathrm{kpc})$ and red: probable association with G29.96-0.02 (near distance, $\sim 6.2 \mathrm{kpc}$ ). The yellow ellipses are the infrared bubbles from Churchwell et al. (2006) discussed in the text.

because it exhibits no clear counterpart in any Hi-GAL band and has no radio counterpart (Deharveng et al. 2010). It is located on the border of a massive, red-supergiant-dominated galactic cluster with a distance upper limit of $7.2 \mathrm{kpc}$ (Clark et al. 2009). Thus N51 could be an old feature. However, the Hi-GAL images show more bubble-like features than what was compiled by Churchwell et al. (2006), opening a new vein for the compilation of these objects.

\subsection{The Galactic context}

Although properties at the scale of our Galaxy require the full coverage of the Hi-GAL survey, we can already underline some global results. To have a general view we first compare the velocity distribution of the sources with the averaged (over the entire Hi-GAL field) spectrum of the ${ }^{13} \mathrm{CO} J=1-0$ emission (Fig. 9). This profile gives information about the molecular complexes and the diffuse emission present along the line of sight. In both fields we can note a prominent narrow component at $9 \mathrm{~km} \mathrm{~s}^{-1}$. This feature is mentioned by Chapin et al. (2008) as a diffuse local component. That this component is seen in both fields with a similar intensity agree with the fact that it is diffuse and local. With a similar velocity as complexes 11 and 12 (Table B.1) we can assign to this feature a distance of $\sim 0.5 \mathrm{kpc}$. We can note that few Hi-GAL sources are found associated with this feature. In both fields we note features corresponding to a large number of Hi-GAL sources, while others are underabundant in Hi-GAL sources with respect to the molecular intensity. This is especially the case for the ${ }^{13} \mathrm{CO} J=1-0$ peak around $81 \mathrm{~km} \mathrm{~s}^{-1}$ in the $l=29^{\circ}$ field and the ${ }^{13} \mathrm{CO} J=1-0$ feature around $35 \mathrm{~km} \mathrm{~s}^{-1}$ in the $l=59^{\circ}$ field. These features correspond to the molecular annulus (Sect. 3.2) and the complex 3 (Table B.2). This indicates that these features are either dominated by diffuse emission or they have a lower star-forming efficiency.

Because velocity diagrams are unable to separate objects with the same $V_{\text {LSR }}$ which still lie at near or far distance, we plot the distance histogram (Fig. 9) and the distribution of sources with regard to the spiral arm structure (Fig. 10) from the recommended distance as discussed in the previous sections.

From Hou et al. (2009) and Russeil et al. (2007) the $l=59^{\circ}$ line of sight should pass close to the Sagittarius arm tangency and then cross the Perseus arm and the Cygnus arm (also called the external arm) at $\sim 9.2$ and $\sim 12.9 \mathrm{kpc}$. Most Hi-GAL sources can be placed in the Sagittarius and Perseus spiral arms (Fig. 10). The few sources around $13.4 \mathrm{kpc}$ can be put in the Cygnus arm. Indeed, the $12.9 \mathrm{kpc}$ distance for the Cygnus arm in the $l=59^{\circ}$ 

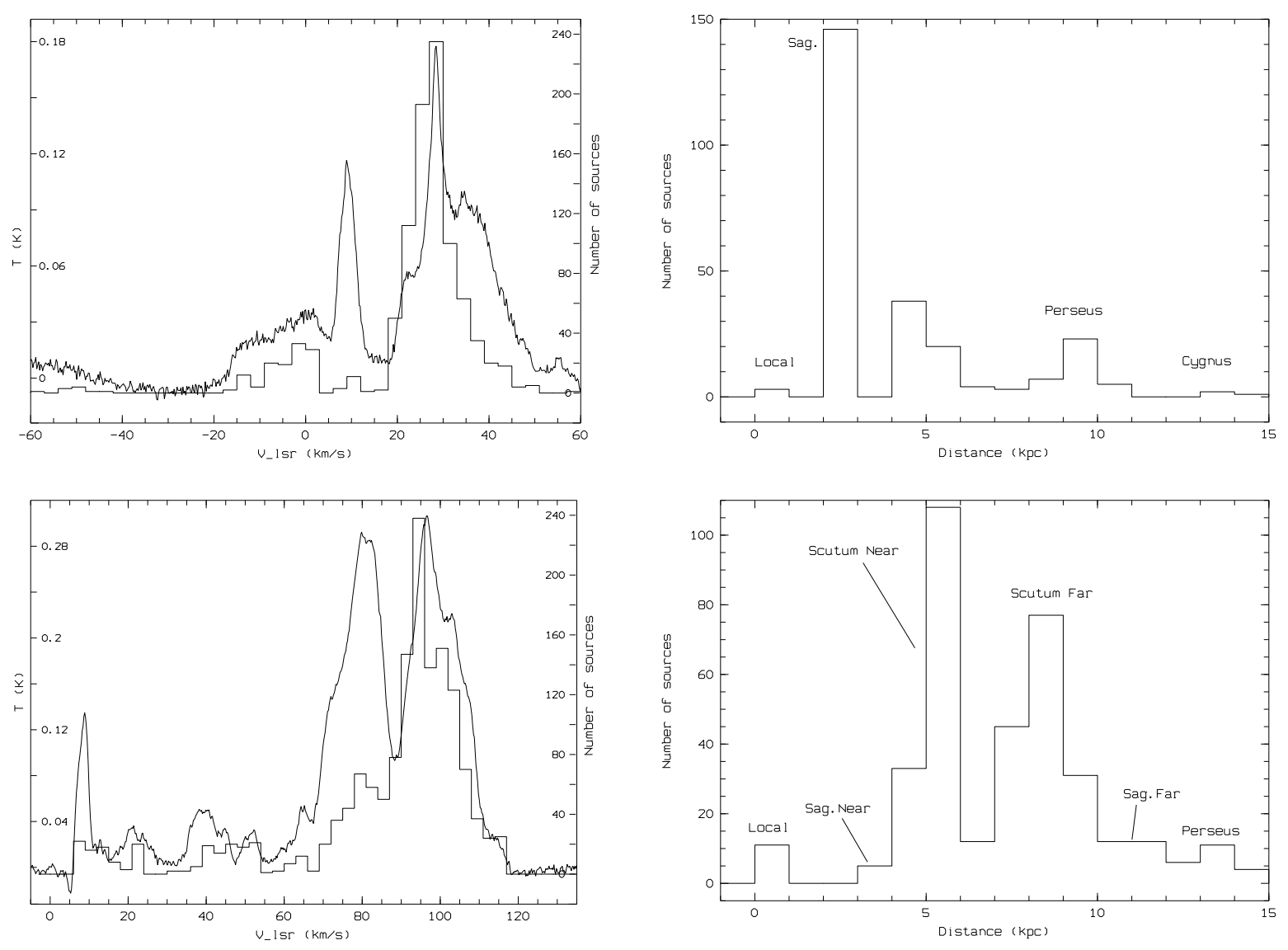

Fig. 9. Histogram with $3 \mathrm{~km} \mathrm{~s}^{-1}$ bins of all sources overplotted on the ${ }^{13} \mathrm{CO} J=1-0$ spectrum averaged on the entire Hi-GAL field for $l=59^{\circ}$ (top left) and $l=30^{\circ}$ (bottom left) and distance histogram $\left(l=59^{\circ}\right.$, top right and $l=30^{\circ}$, bottom right).

direction is established from spiral arm fitting only because no star-forming tracer was previously detected.

The $l=30^{\circ}$ line of sight is more complicated because it points towards crowded parts of the Galactic plane. From a comparison with the Hou et al. (2009) spiral arms model and the similarly to the distribution of H II regions (Anderson et al. 2009) and molecular clouds (Roman-Duval et al. 2009), it appears that most of the sources are located in the Scutum-Crux arm, while few sources can be put on the far side of the Sagittarius and Perseus arms (Fig. 10).

It can be seen from Fig. 10 that both lines of sight show several sources falling in the inter-arm regions whatever the spiral arm model. Figure 9 (right) clearly shows the different spiral arm structure expected along the line of sight. For the $l=59^{\circ} \mathrm{di}-$ rection in addition to the expected spiral arms a distinct feature around $5 \mathrm{kpc}$ is noted. It is associated with the $35 \mathrm{~km} \mathrm{~s}^{-1}{ }^{13} \mathrm{CO}$ feature. Its status as an isolated inter-arm star-forming cloud or a large scale feature (arm or spur) will be clarified through upcoming Hi-GAL observation of the adjacent fields. For the $l=30^{\circ}$ direction we can conclude that the molecular annulus is located in the near part of Scutum arm.

The quite good correlation of Hi-GAL sources with spiral arms confirm that Hi-GAL sources are mainly linked to starformation. Hi-GAL data therefore appear to be a good tracer for studying and understanding the large-scale structure of our Galaxy.

A particularly interesting study that will be addressed with Hi-GAL is the dependence of different quantities related to star formation on galactocentric distance. One of these is star-formation efficiency. A rough estimate of this quantity can be obtained by comparing the surface density of Hi-GAL sources (assuming these are the best representatives of young, star-forming stars and star clusters) with the surface density of molecular gas in the Milky Way. In general one can say that the more (free) gas there is per source, the less efficient is the process of conveying gas into stars, and hence the less efficient is the star formation. Figure 11 shows this comparison, as a very early attempt to identify a possible trend. The histogram of the HiGAL source distribution (right axis) is overplotted on the molecular gas surface density (left axis), both plotted as a function of galactocentric distance, i.e. averaged in azimuthal angle. The histogram of Hi-GAL sources is a natural outcome of the estimate of kinematic distances, because the galactocentric distance does not suffer from the near / far ambiguity. The molecular gas distribution is taken from Blitz (1996) and was derived by converting the azimuthally averaged distribution of CO in the Milky Way (see also e.g. Bloeman 1986; Strong \& Mattox 1996).

In Fig. 11 it is possible to estimate that in the inner Galaxy there is about 100 times less gas per Hi-GAL source than in the outer galaxy, indicating that star formation must be more efficient in the inner galaxy. This remains a first estimate and it is not meant to settle the issue. In particular the completeness of the source catalogues, especially for the outer Galaxy, must be established to give solid ground to the plot. Also, a precise assessment of the evolutionary stage of Hi-GAL sources will need to be undertaken in order not to contaminate the estimate with more evolved sources. Finally, data from more Hi-GAL tiles have to 


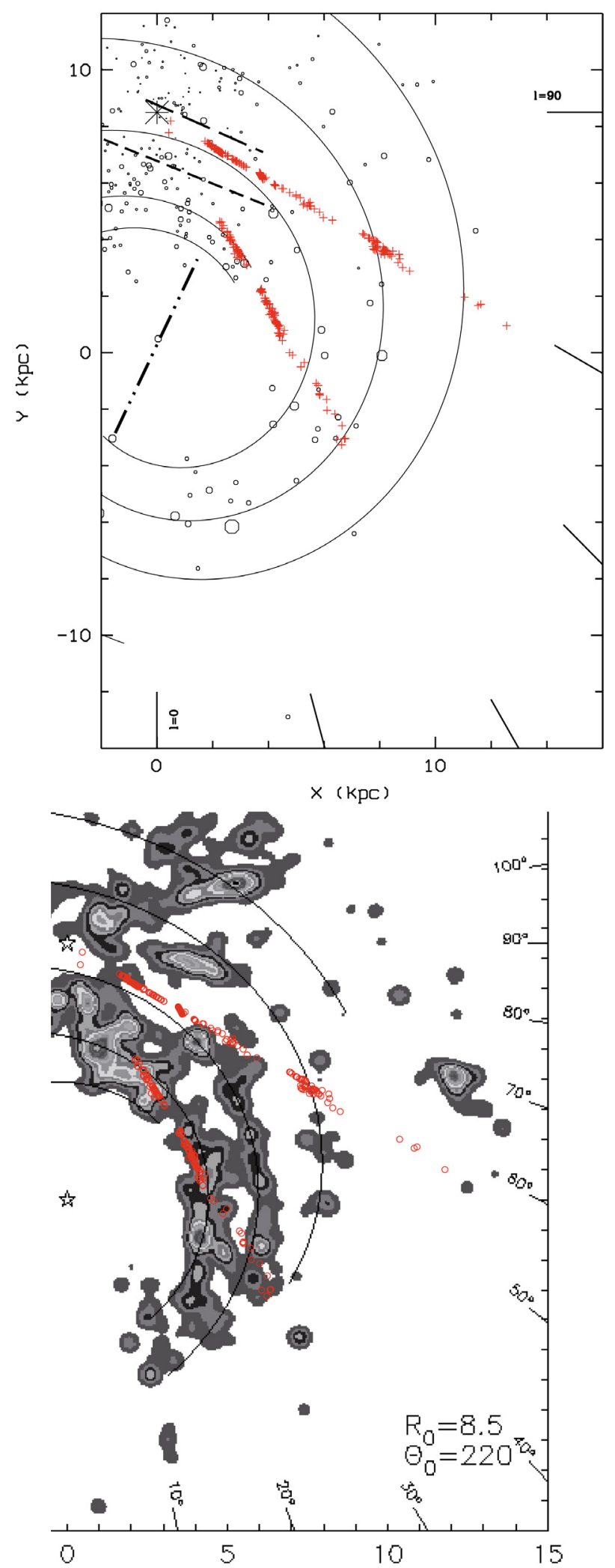

Fig. 10. Hi-GAL sources (red symbols), with unique velocity determination, overplotted on spiral arm models. For both models the Sun is at $8.5 \mathrm{kpc}$ and its circular velocity $220 \mathrm{~km} \mathrm{~s}^{-1}$. Top: model from Russeil et al. (2007) based on the Brand \& Blitz (1993) rotation curve. The circles are the star-forming complexes. Bottom: model from Hou et al. (2009) based on a rotation curve similar to that of Brand \& Blitz (1993). Star symbols locate the Sun and the Galactic Centre. The greyscale features represent the density distribution of $\mathrm{H}$ II and massive molecular clouds.

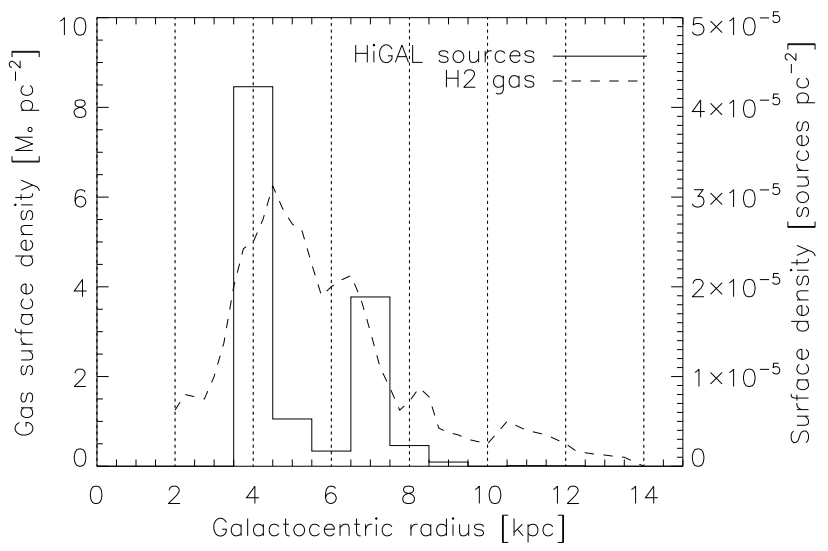

Fig. 11. Comparison of Hi-GAL source surface density (histogram and right $y$-axis) with $\mathrm{H}_{2}$ gas surface density (dashed line and left $y$-axis from Blitz 1996) in galactocentric radius. The ratio between the two quantities is a proxy for star-formation efficiency: the more $\mathrm{H}_{2}$ gas per Hi-GAL source, the less efficient is the star formation. From the plot it is possible to estimate that in the outer Galaxy there is about 100 times more gas per Hi-GAL source than in the inner Galaxy (galactic ring, around $4-5 \mathrm{kpc}$ from the Galactic centre), supporting the idea that star formation is more efficient in the inner Galaxy.

be analysed to probe a larger range of galatocentric radii than is possible with only the two tiles centred at $l=30^{\circ}$ and $l=59^{\circ}$.

\section{Conclusions}

We demonstrate that from a multiwavelength approach we are able to solve the near/far distance ambiguity problem for sources inside to the solar circle. The kinematic distance is then established for all sources with measured velocities, while for sources which are associated with $\mathrm{H}$ II regions the stellar distance has been adopted.

The main points to emphasize are:

1. Thanks to the pipeline, $93 \%$ of the 2678 compact sources have been assigned a radial velocity. Excluding the velocity of the sources with no distance ambiguity problem (sources outside the solar circle), this leads to $89 \%$ of the sources for which the pipeline has been able to determine their distances.

2. It is important to conduct a systematic follow-up of the sources in $\mathrm{NH}_{3}$ to have a unique velocity measurement. Indeed, lots of sources exhibit double or multiple $\mathrm{CO}$ velocity components.

3. It clearly appears throughout this paper that multiwavelength information and multiple techniques are required to solve the distance ambiguity.

4. The Hi-GAL sources are mainly located in spiral arms, but some sources seem to fall in inter-arm regions. The distance, the distance uncertainty, and/or the nature of these interarm sources must be clarified before comparing them with sources in the arms.

5. Some Hi-GAL sources are found to trace parts of spiral arms where up to now no other star-formation tracers were detected.

Because we can assume that Hi-GAL sources are mainly linked to star formation, Hi-GAL data are a good tracer for studying the large-scale structure in the disk of our Galaxy. Up to now the spiral arm models are obtained by fitting the spiral arm tracer with 
weighting factors based on the excitation parameter of $\mathrm{H}$ II regions (e.g. Russeil 2003) or the mass of GMCs (Hou et al. 2009) or by finding the tangential direction to the arms from $\mathrm{CO}$ or near-infrared longitude profiles (e.g. Benjamin 2008; Drimmel 1986). We can expect to trace the spiral arms with infrared fluxes as weighting factors and/or to trace far-infrared profiles to state more precisely the arm tangent directions.

From the technical point of view we plan to develop a semiautomatic process for the distance recommendation determination to systematically apply it for the incoming Hi-GAL fields. In addition, as illustrated in this paper, the Hi-GAL survey will detect lots of sources for which the distance, hence the velocity, will have to be established. In this way important molecular observational efforts are in progress by the Hi-GAL members to determine the velocity of all these new objects.

Acknowledgements. We thank C. Persson for her active participation in the $\mathrm{NH}_{3}$ observations. We thank N. Wright from the IPHAS project for processing the $\mathrm{H} \alpha$ mosaic image of Sh2-86. We thank the referee, Professor M. Burton, for his useful comments and remarks. The paper is partly based on observations with the Medicina telescope operated by INAF - Istituto di radioastronomia and Onsala telescope operated by Swedish National Facility for Radio Astronomy. Part of this work was supported by the ANR (Agence Nationale pour la Recherche) project "PROBeS", number ANR-08-BLAN-0241. Part of this work was done thanks to SIMBAD at CDS. The VGPS is supported by a grant from the Natural Sciences and Engineering Research Council of Canada and from the US National Science Foundation. The National Radio Astronomy Observatory is a facility of the National Science Foundation operated under cooperative agreement by Associated Universities, Inc.

\section{References}

Anderson, L., \& Bania, T. 2009, ApJ, 690, 706

Anderson, L., Bania, T., Jackson, et al. 2009, ApJS, 181, 255

Baba, J., Asaki, Y., Makino, J., et al. 2009, ApJ, 706, 471

Benjamin, R. 2008, Massive Star Formation: Observations Confront Theory, ed.

H. Beuther, H. Linz, \& T. Henning, ASP Conf. Ser., 387, 375

Benjamin, R. A., Churchwell, E., Babler, B. L., et al. 2003, PASP, 115, 953

Billot, N., Noriega-Crespo, A., \& Carey, S. 2010, ApJ, 712, 812

Bovy, J., Hogg, D. W., \& Rix, H.-W. 2009, ApJ, 704, 1704

Blitz, L. 1997, ed. W. B. Latter, S. J. E., Radford, \& P. R. Jewell, IAU Symp., 170,11 ,

Bloemen, J. B. 1986, BAAS, 18, 929

Brand, J., \& Blitz, L. 1993, A\&A, 275, 67

Burton, W. 1971, A\&A, 10, 76

Busfield, A. L., Purcell, C. R., Hoare, M. G., et al. 2006, MNRAS, 366, 1096

Chapin, E. L., Ade, P., Bock, J., et al. 2008, ApJ, 681, 428
Churchwell, E., Povich, M. S., Allen, D., et al. 2006, ApJ, 649, 759 Clark, J. S., Negueruela, I., Davies, B., et al. 2009, A\&A, 498, 109 Clements, D. L., Rigby, E., Maddox, S., et al. 2010, A\&A, 518, L8 Dame, T., Elmegreen, B., Cohen, R., et al. 1986, ApJ, 305, 892

Deharveng, L., Schuller, F., Anderson, L. D., et al. 2010, A\&A, 523, A6 Drimmel, R. 2000, A\&A, 358, L13

Ehlerová, S., \& Palous, J. 2005, A\&A, 437, 101

Elia, D., Schisano, E., Molinari, S., et al. 2010, A\&A, 518, L97

Fich, M., Dahl, G., \& Treffers, R. 1990, AJ, 99, 622

Fich, V. L., Reid, M. J., Wilner, D. J., et al. 2003, ApJ, 587, 701

Forbes, D. 1989, A\&ASS, 77, 439

Guetter, H. H. 1992, AJ, 103,197

Heiles, C. 1979, ApJ, 229, 533

Hou, L., Han, J., \& Shi, W. 2009, A\&A, 499, 473

Hoyle, F., Shanks, T., \& Tanvir, N. 2003, MNRAS, 345, 269

Jacq, T., Despois, D., \& Baudry, A. 1988, A\&A, 195, 93

Jackson, J. M., Bania, T. M., Simon, R., et al. 2002, ApJ, 566,81

Jackson, J. M., Rathborne, J. M., Shah, R. Y., et al. 2006, ApJS, 163, 145

Jackson, J. M., Finn, S., Rathborne, M., et al. 2008, ApJS, 680, 349

Kim, K., \& Koo, B. 2001, ApJ, 549, 979

Knapp, G. R. 1974, AJ, 79, 527

Kolpack, M., Jackson, J., \& Bania, T. 2003, ApJ, 582, 756

Kuchar, T., \& Bania, T. 1994, ApJ, 436, 117

Marshall, D. J., Robin, A. C., Reylé, C., et al. 2006, A\&A, 453, 635

Marshall, D. J., Joncas, G., \& Jones, A. P. 2009, ApJ, 706, 727

Massey, P., johnson, K. E., \& Degoia-Eastwood, K. 1995, ApJ, 454, 151

McMillan, P. \& Binney, J. 2010, MNRAS, 402, 934

Molinari, S., Swinyard, B., Bally, J., et al. 2010, A\&A, 518, L100

Pandian, J., Momjian, E., \& Goldsmith, P. 2008, A\&A, 486, 191

Penã, J., Garcia-Cole, A., Hobart, M., et al. 2003, Rev. Mex. AA, 39, 171

Pratap, P., Megeath, S. T., \& Bergin, E. A. 1999, ApJ, 517, 799

Quireza, C., Robert, T., Dana, S., et al. 2006, ApJS, 165, 338

Reid, M., Menten, K., Zheng, X., et al. 2009, ApJ, 700, 137

Robin, A. C., Reylé, C., DerriẤlre, S., et al. 2003, A\&A, 409, 523

Roman-Duval, J., Jackson, J. Heyer, M., et al. 2009, ApJ, 699, 1153

Russeil, D., Adami, C., \& Georgelin, Y. 2007, A\&A, 470, 161

Russeil, D. 2003, A\&A, 297, 133

Schneider, N., Bontemps, S., Simon, R., et al. 2010, A\&A, accepted [arXiv: 1001.2453]

Schuller, F., Menten, K., Contreras, Y., et al. 2009, A\&A, 504, 415

Sewilo, M., Watson, C., Araya, E., et al. 2004, ApJS, 154, 553

Simon, R., Rathborne, J., Shah, R., et al. 2006, ApJ, 653, 1325

Solomon, P., Rivolo, A., Barrett, J., et al. 1987, ApJ, 319, 730

Stark, A., \& Brand, J. 1989, ApJ, 339, 763

Stil, J. M., Taylor, A. R., Dickey, J. M., et al. 2006, AJ, 132, 1158

Strong, A. W., \& Mattox, J. R. 1996, A\&A, 308, 21

Skrutskie, M. F., Cutri, R. M., Stiening, R., et al. 2006, AJ, 131, 1163

Watson, C., Araya, E., Sewilo, M., et al. 2003, ApJ, 587, 714

Xu, Y., Reid, M., Menten, K., et al. 2009, ApJ, 693, 413

Zavagno, A., Anderson, L. D., Russeil, D., et al. 2010, A\&A, 518, L101 


\section{Appendix A: Figures}

Additional figures are presented in this Appendix.

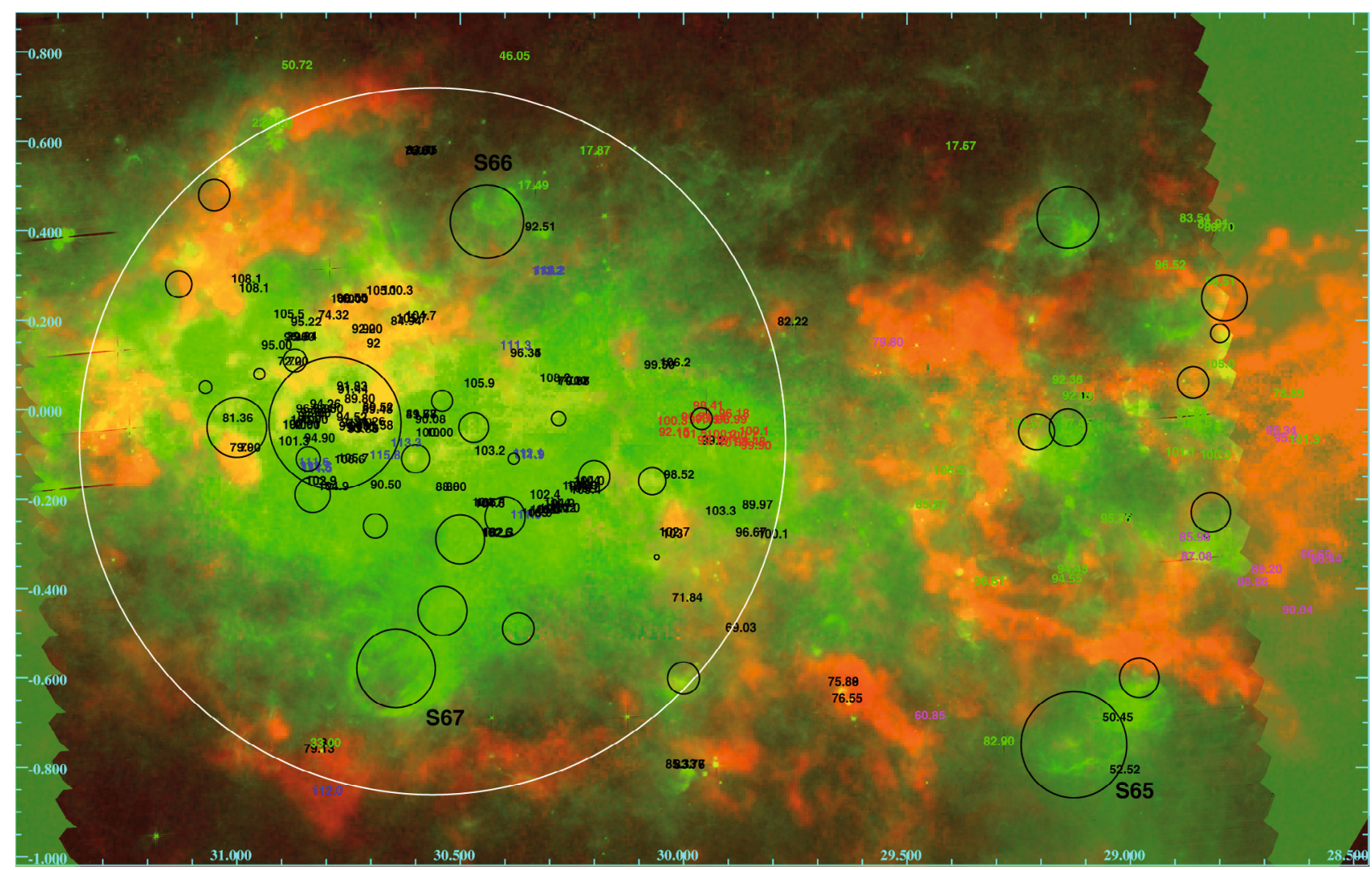

Fig. A.1. View of the $l=29^{\circ}$ field (the coordinates are galactic coordinates). In red is given the ${ }^{13} \mathrm{CO}$ emission at velocities between 75 and $85 \mathrm{~km} \mathrm{~s}^{-1}$ showing the possible molecular annulus (white circle) and in green the PACS $70 \mu \mathrm{m}$ emission. Black circles show H II regions (the size of the circles corresponds to the physical size, see e.g. Anderson et al. 2009) with known velocity. The colours of the source velocities correspond to: black: probable association with W43 for sources around $94 \mathrm{~km} \mathrm{~s}^{-1}(\sim 5.8 \mathrm{kpc})$ and association with Sh2-65 for sources around $51 \mathrm{~km} \mathrm{~s}^{-1}(3.3 \mathrm{kpc})$, blue: tangent distance $(\sim 7.4 \mathrm{kpc})$, green: far or tangent distance (e.g. $\sim 12.4 \mathrm{kpc}$ for sources $\left.\sim 20 \mathrm{~km} \mathrm{~s}\right)^{-1}, \sim 7.5 \mathrm{kpc}$ for sources $\left.\sim 95 \mathrm{~km} \mathrm{~s}^{-1}\right)$, magenta: near distance $(\sim 5.3 \mathrm{kpc})$ and red: probable association with G29.96-0.02 (near distance, $\sim 6.2 \mathrm{kpc}$ ).

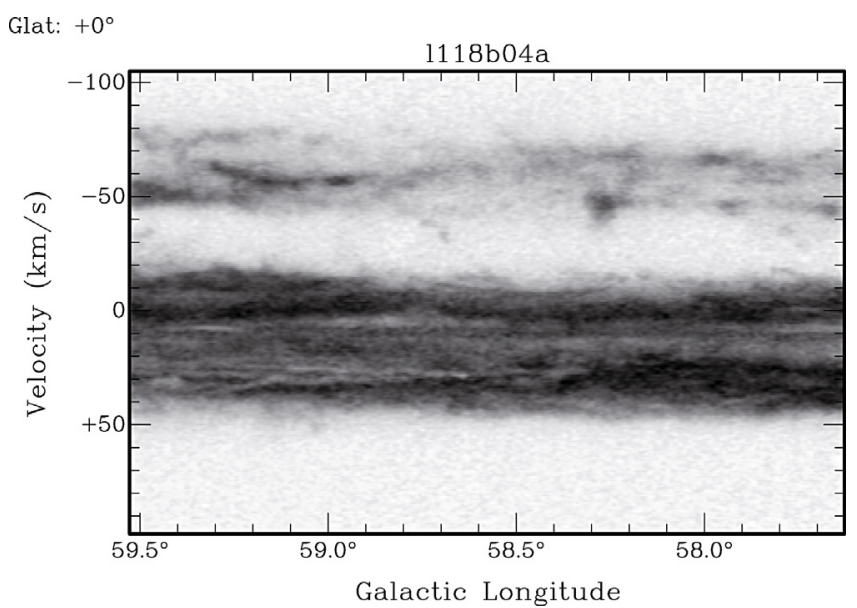

Fig. A.2. VGPS-HI longitude-velocity plot at the galactic latitude $0^{\circ}$. 


\section{Appendix B: Tables}

The columns of the following two tables are:

Column 1: A complex identification number is given, the near/far/tangent distances (kpc) are also given from the mean molecular velocity and mean $1, \mathrm{~b}$ coordinates of the complex. If it is not possible to calculate the distance or if there is no distance ambiguity, only the valid distance is given. The near, far, or tangent choice for the complex is also indicated (if not indicated, the distance in Col. 2 is adopted).

Column 2: The stellar (or maser parallax) distance $(\mathrm{kpc})$.

Column 3: The optical name of associated H II region(s).

Column 4: Galactic coordinates of the radio H II regions and identification of molecular clouds. "Wat", "SW", "A" correspond to radio sources listed mainly in Watson et al. (2003), Sewilo (2004) and Anderson et al. (2009). The molecular information is from Dame et al. (1986) for "GMC", from Roman-Duval et al. (2009) for "GRSMC" clouds, from Solomon et al. (1987) for "Sol" clouds, from Jacq et al. (1988) for "Bx" clouds and from Simon et al. (2006) and Jackson et al. (2008) for "IRDC". For "GMC" and "Bx" clouds the radius and velocity FWHM of the cloud is given in brackets. Note that the typical radius and velocity FWHM of "GRSMC" clouds are $0.35 \pm 0.12$ degrees and $3.57 \pm 1.21 \mathrm{~km} \mathrm{~s}^{-1}$. These values are lower than the typical values for GMC.
Column 5: $\mathrm{H} \alpha$ velocity. When different values are found in the literature, they are given separated by a semicolon.

Column 6: Radio recombination-line velocity. When different values are found in the literature, they are given separated by a semicolon. If several velocities (along the line of sight) are measured they are separated by a backslash.

Column 7: Molecular line velocities (CO and/or CS). When different values are found in the literature, they are given separated by a semicolon. If several velocities (along the line of sight) are measured, they are separated by a backslash.

Column 8: $\mathrm{H}_{2} \mathrm{CO}$ or $\mathrm{OH}$ absorption line velocities (rounded to the nearest value) detected along the line of sight of the source. When different values are found in the literature, they are given separated by a semicolon. If several velocities (along the line of sight) are measured, they are separated by a backslash.

Column 9 gives the near/far/tangent solution mainly from $\mathrm{HI}$ emission/absorption or HI self-absorption methods and references.

$\mathrm{N}$ means near, $\mathrm{F}$ means far, $\mathrm{T}$ means tangent. When two solutions are proposed in brackets the first comes from the HI emission/absorption method, the second from the HI selfabsorption method (Anderson \& Bania 2009). For sources where the two methods disagree, the favoured one by Anderson \& Bania (2009) is marked with an asterisk. 


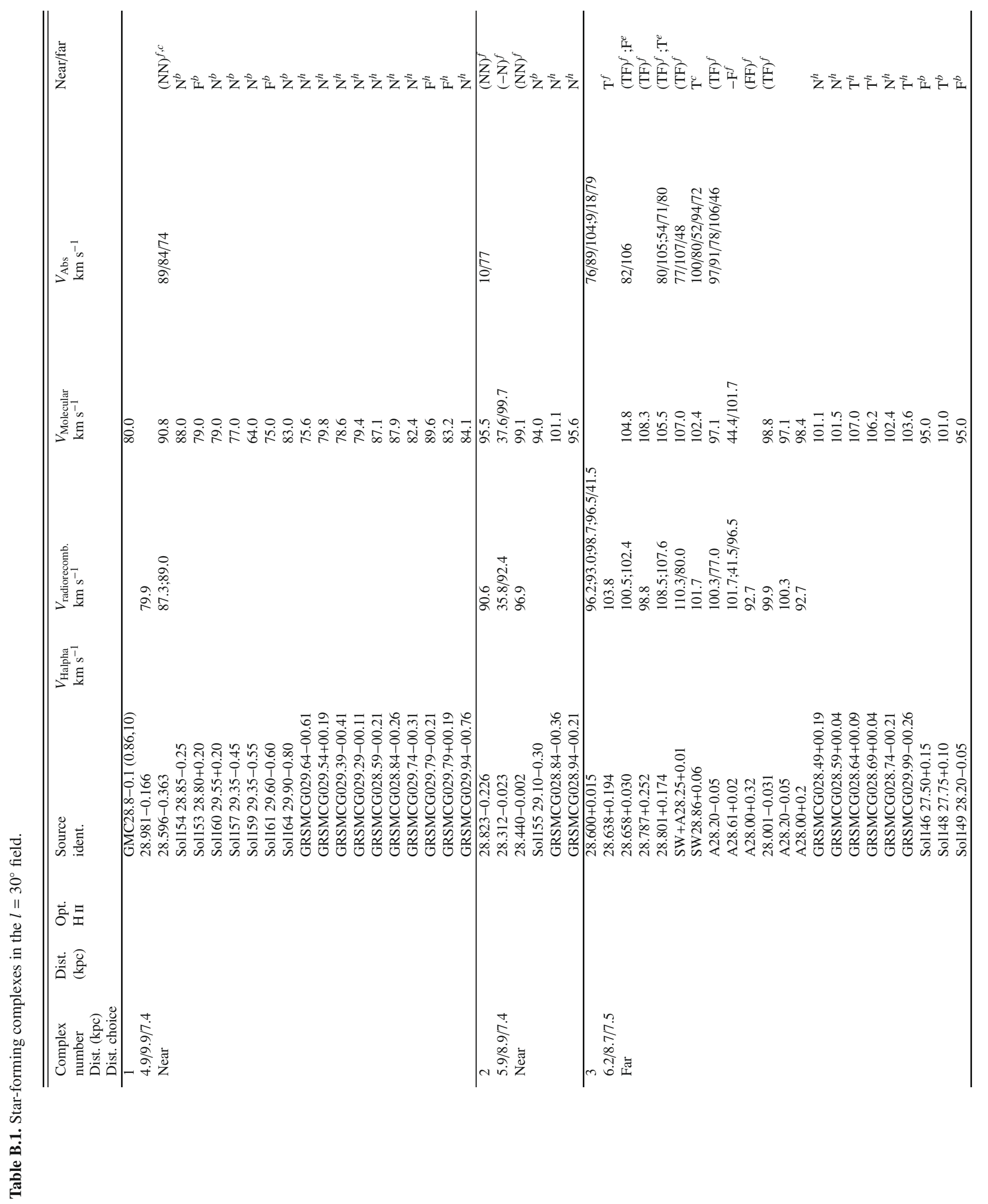




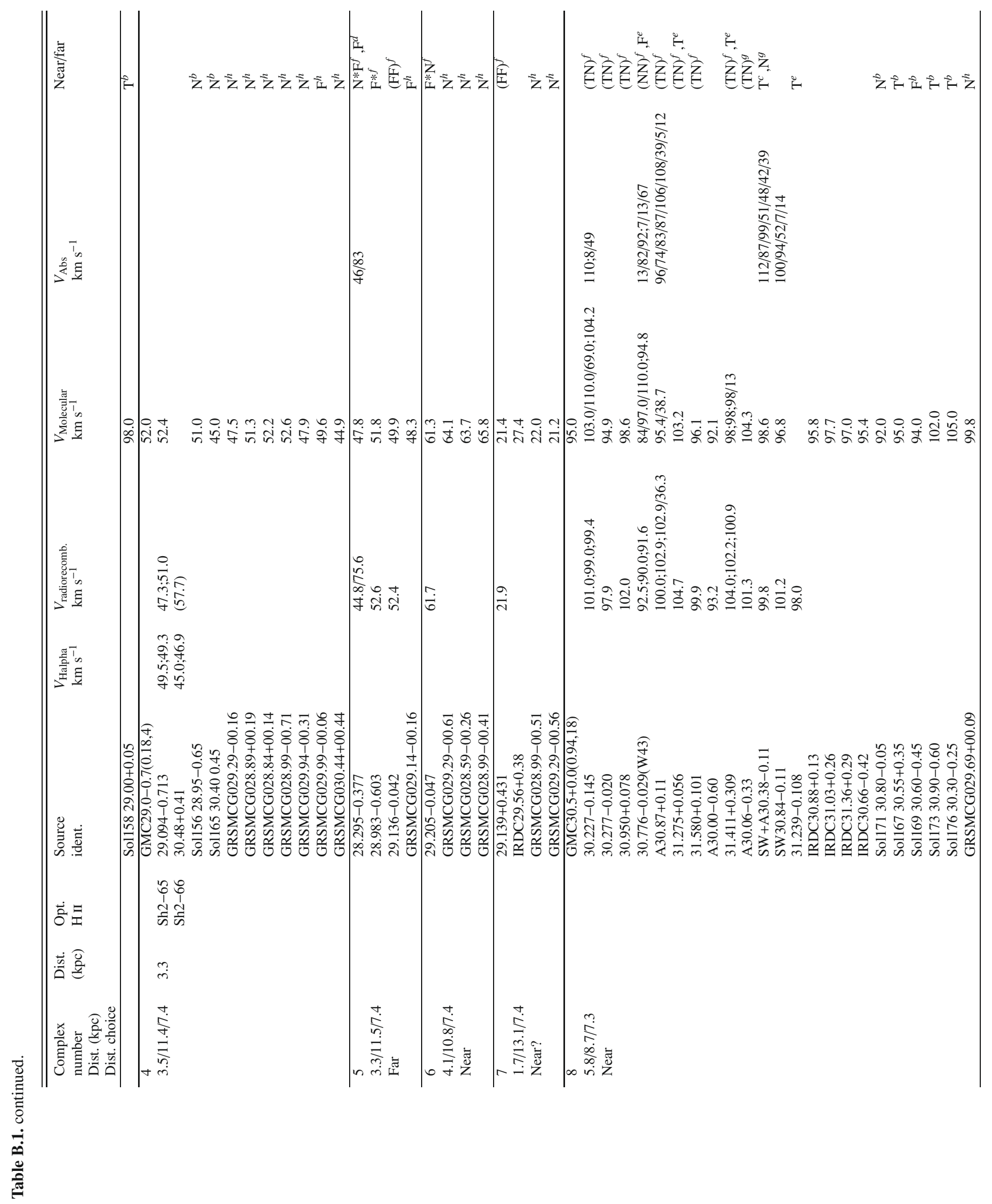




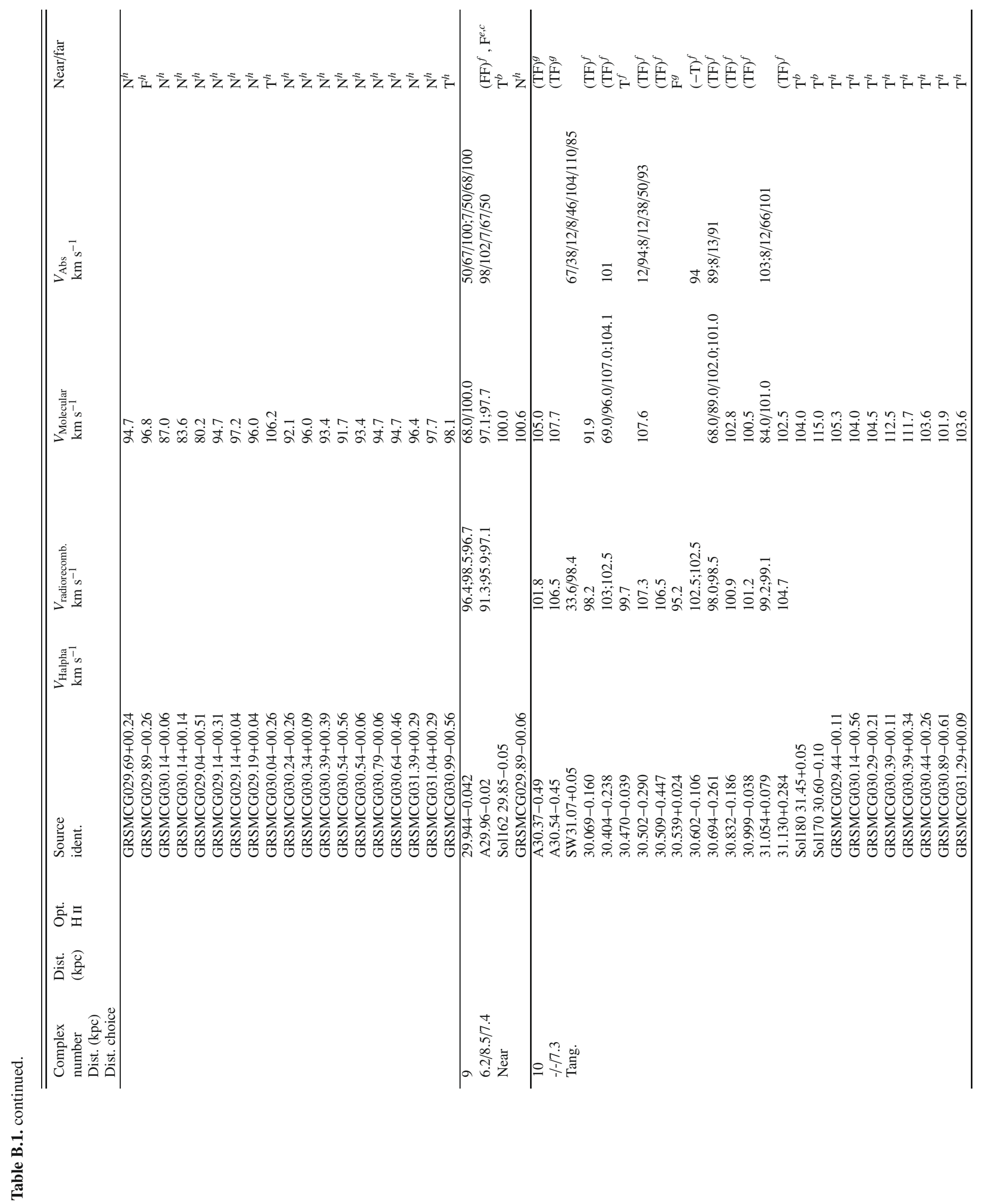




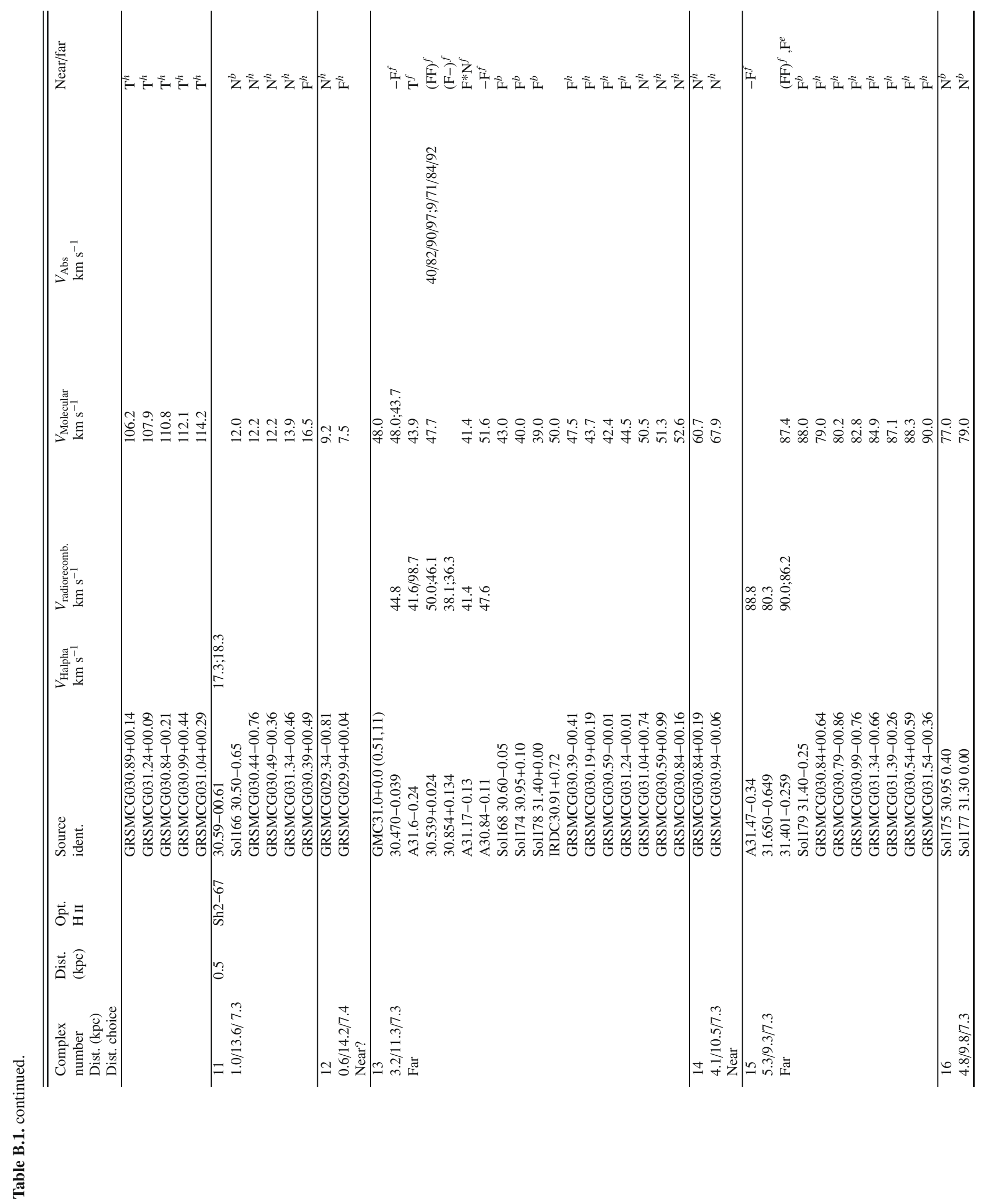




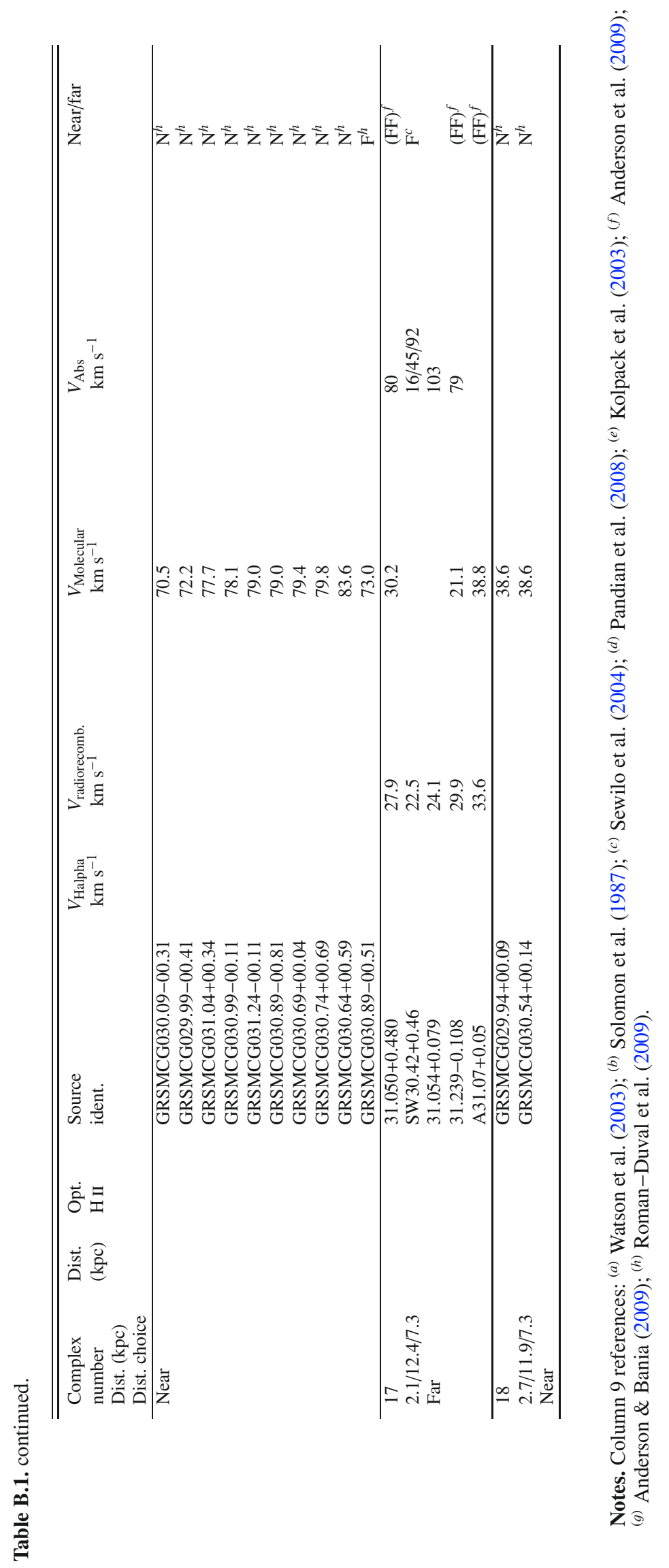


Table B.2. Star-forming complexes in the $l=59^{\circ}$ field.

\begin{tabular}{|c|c|c|c|c|c|c|c|c|}
\hline $\begin{array}{l}\text { Complex } \\
\text { number } \\
\text { Dist. }(k p c) \\
\text { Dist. choice }\end{array}$ & $\begin{array}{l}\text { Dist. } \\
(\mathrm{kpc})\end{array}$ & $\begin{array}{l}\text { optical } \\
\text { H II reg. }\end{array}$ & $\begin{array}{l}\text { source } \\
\text { ident. }\end{array}$ & $\begin{array}{l}V_{\text {Halpha }} \\
\mathrm{km} \mathrm{s}^{-1}\end{array}$ & $\begin{array}{l}V_{\text {radiorecomb. }} \\
\mathrm{km} \mathrm{s}^{-1}\end{array}$ & $\begin{array}{l}V_{\text {Molecular }} \\
\mathrm{km} \mathrm{s}^{-1}\end{array}$ & $\begin{array}{l}V_{\mathrm{Abs}} \\
\mathrm{km} \mathrm{s}^{-1}\end{array}$ & Near/far \\
\hline & & & $57.541-0.276$ & & 2.8 & & & \\
\hline $0.5 / 8.7 / 4.6$ & & & Bx N 56.8+0.0(0.63,4.8) & & & 8.8 & & \\
\hline Far & & & Wat57.55-0.27 & & 6.3 & & $2 / 10 / 31$ & $\mathrm{~F}^{a, c}$ \\
\hline $\begin{array}{l}2 \\
-/ 10 /-\end{array}$ & & & $\begin{array}{l}59.796+0.237 \\
\text { Bx W 57.2+0.0(0.61,8.2) }\end{array}$ & & $-6 ;-2.8$ & -7.0 & 42 & $\mathrm{~F}^{c}$ \\
\hline 3 & & & Wat58.77+0.65 & & 30.0 & & 33 & \\
\hline $\begin{array}{l}-/-/ 4.3 \\
\text { Tang. }\end{array}$ & & & Wat59.60+0.92 & & 46.4 & & 37 & \\
\hline 4 & 2.3 & Sh2-86 & $59.529-0.181$ & $26.4 ; 27.9$ & 29.4 & 26.8 & & $\mathrm{~F}^{c}$ \\
\hline $2.3 / 6.2 / 4.2$ & & Sh2-87 & $60.888-0.127$ & 17.9 & 18.6 & 22.7 & & \\
\hline & & Sh2-88 & $61.470+0.90$ & $19.3 ; 19.8$ & 27.3 & 22.9 & $22 / 6$ & $\mathrm{~T}^{a}$ \\
\hline & & Sh2-89 & $62.941+0.084$ & 12.7 & 19.2 & 25.6 & & \\
\hline & & Sh2-90 & $63.176+0.460$ & $22.5 ; 18.0$ & $15.3 ; 19.4$ & 22.2 & & \\
\hline & & & Вх О $60.0+0.0(0.31,9)$ & & & 24.5 & & \\
\hline & & & Sol245 59.30-0.20 & & & 28.0 & & $\mathrm{~N}^{b}$ \\
\hline & & & Sol246 60.00+0.10 & & & 22.0 & & $\mathrm{~F}^{b}$ \\
\hline & & & Sol247 60.90-0.10 & & & 23.0 & & $\mathrm{~F}^{b}$ \\
\hline & & & Sol248 61.50+0.10 & & & 22.0 & & $\mathrm{~F}^{b}$ \\
\hline & & & Sol249 $63.10+0.40$ & & & 20.0 & & $\mathrm{~F}^{b}$ \\
\hline & & & Wat59.36-0.21 & & 26.6 & & 29 & \\
\hline & 2.16 & & Mas59.78+0.06 & & & 27.0 & & \\
\hline
\end{tabular}

Notes. Column 9 references: ${ }^{(a)}$ Watson et al. (2003) ; ${ }^{(b)}$ Solomon et al. (1987) ; ${ }^{(c)}$ Kuchar \& Bania (1994) . 